\title{
Ecological Performance of Multifunctional Pesticide Tolerant Strains of Mesorhizobium Sp. In Chickpea With Recommended Pendimethalin, Ready-mix of Pendimethalin and Imazethpyr, Carbendazim and Chlorpyrifos Application
}

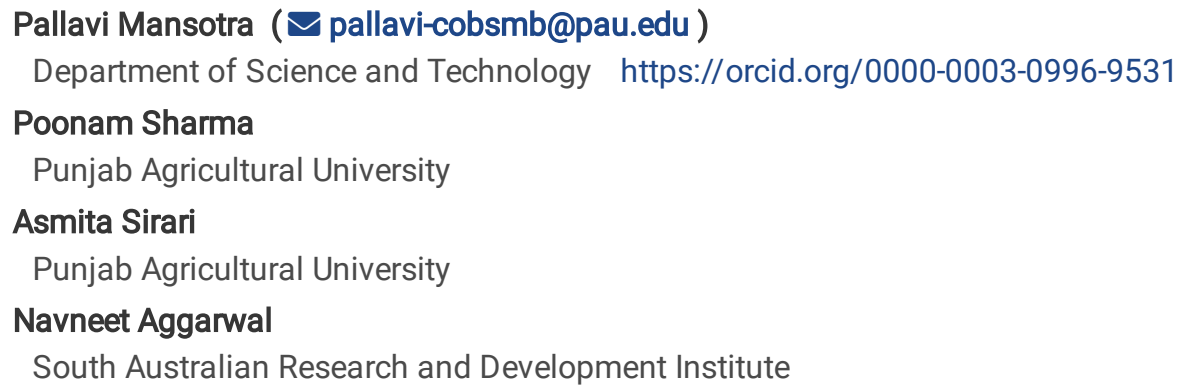

\section{Research Article}

Keywords: Multifunctional pesticide tolerant mesorhizobia, Dual inoculation, Plant growth, SNF, Grain yield, Chickpea

Posted Date: August 6th, 2021

DOI: https://doi.org/10.21203/rs.3.rs-717985/v1

License: (c) (i) This work is licensed under a Creative Commons Attribution 4.0 International License. Read Full License

Version of Record: A version of this preprint was published at Archives of Microbiology on January 1st, 2022. See the published version at https://doi.org/10.1007/s00203-021-02628-5. 


\section{Abstract}

The present study was designed to screen the Mesorhizobium strains (50) for tolerance in four recommended pesticides for chickpea. It was followed by in-vitro development of robust pesticide tolerant strains by growth in pesticides amended media over several generations. Further, verification of the multifunctional traits of pesticide tolerant mesorhizobia under pesticide stress was conducted invitro. Among different pesticides, significantly high tolerance in Mesorhizobium strains was observed with recommended doses of pendimethalin (37\%) and ready-mix (36\%) followed by chlorpyrifos (31\%) and carbendazim (30\%), on an overall basis. Based on multifunctional traits, Mesorhizobium strains viz. MR2, MR17 and recommended MR33 were the most promising. Ecological performance of the potential Mesorhizobium strains alone and in dual-inoculation with recommended PGP rhizobacterium strain RB-1 (Pseudomonas argenttinensis JX239745.1) was further analyzed in field following standard pesticide application in PBG-7 and GPF-2 chickpea varieties for two consecutive rabi seasons (2015 and 2016). Dual-inoculant treatments; recommended RB-1+MR33 (4.1\%) and RB-1+MR2 (3.8\%) significantly increased the grain yield over Mesorhizobium alone treatments viz MR33 and MR2, respectively. Grain yield in PBG7 variety was significantly affected $(7.3 \%)$ by the microbial inoculant treatments over GPF2 variety. Therefore, the potential pesticide tolerant strains MR2 and MR33 can be further explored as compatible dual-inoculants with recommended RB-1 for chickpea under environmentally stressed conditions (pesticide application) at multiple locations. Our approach using robust multifunctional pesticide tolerant Mesorhizobium for bio-augmentation of chickpea with might be helpful in the formulation of effective bio-inoculants consortia in establishing successful chickpea-Mesorhizobium symbiosis.

\section{Introduction}

Chickpea is the most important rabi pulse crop of India with an increase in production from 6.5 million tonnes to 10.3 million tonnes and grain yield from 630 to $935 \mathrm{Kg} \mathrm{ha}^{-1}$ during the past 35 years (Singh et al. 2018). Inoculation of chickpea seeds with a selected strain of Mesorhizobium sp. is under wide practice to improve the yield by enhancing root nodulation and $\mathrm{N}$ uptake of the plant. This helps in reducing the dependency on chemical fertilizers (Brigido et al. 2017). Co-inoculation of plant growth-promoting (PGP) bacteria with Rhizobium synergistically improves the legume growth, symbiotic nitrogen fixation (SNF), yield, and overall plant's performance by absorption of nitrogen, phosphorus and other macro-and micronutrients (Gopalakrishnan et al. 2015). Therefore, identification and manipulation of their relationships with PGP bacteria might fulfill modern demands of agricultural, economic, social, and environmental sustainability (Fatnassi et al. 2015). Chickpea seeds are susceptible to varied fungal pathogens (Ascochyta blight and Botrytis grey mold), insect, termites and weeds. Under a recommended strategy of maximizing productivity, farmers often treat the seeds with fungicides (carbendazim) followed by insecticides (chlorpyrifos) to protect them from seed-borne pathogens and then inoculate them with a nitrogen $\left(\mathrm{N}_{2}\right)$ fixing strain to use the natural resources. Also, there are two recommended herbicides viz. pre-emergence (pendimethalin) and pre-plant (ready-mix which is a combination of pendimethalin + imazethapyr) herbicides for controlling broadleaved weeds in chickpea (Anonymous 2013). However, the problems arise when we use chemical pesticide treatments in conjunction with Mesorhizobium/Mesorhizobium + rhizobacterium as bio-fertilizer. Adverse effects of pesticides on legume-rhizobia symbiosis include inhibition of cell division and nodule formation, limiting carbohydrate supply to the nodules, blocking the initial attachment of complementary rhizobia to lectins by protecting recognition sites present on root hairs and inhibition of symbiotic signaling between host plant and rhizobia (Meena et al. 2020). One of the major constraints faced in Rhizobium inoculant development technology is the selection of isolates that are symbiotically efficient and adapted to the local environmental conditions loaded with various types of stress (Porter et al. 2019). Previous studies have largely focussed on the effects of few pesticides in a limited number of mesorhizobia on SNF in chickpea. However, there is scarce information on pesticide tolerant mesorhizobia in chickpea. The present investigation was designed to screen indigenous Mesorhizobium strains for intrinsic tolerance to recommended pesticides followed by verification of their in-vitro multifunctional traits under pesticide stress. Further, the robust strains of Mesorhizobium with multifunctional traits were explored for ecological performance on plant growth, SNF and yield in chickpea crop raised with the recommended application of pesticides under field conditions.

\section{Materials And Methods}

\subsection{Procurement and purity of reference cultures}

Seventy-five strains of Mesorhizobium sp. including recommended cultures of MR33 (Mesorhizobium cicersp. LGR33) and PGP rhizobacterium strain RB-1 (Pseudomonas argenttinensis JX239745.1) were procured from the culture collection of Pulses Research Laboratory, Department of Plant Breeding and Genetics, PAU, Ludhiana. The purity of cultures was tested using the Gram staining technique (Ouma et al. 2016). Further, for differentiation of Mesorhizobium from Agrobacterium and other contaminants, different 
confirmatory tests were performed on the Hoffer's alkaline (Ansari et al., 2014), the ketolactose, (Finer et al. 2016), the congo red yeast extract mannitol agar (CRYEMA) and the glucose peptone agar media (Gaur and Sen 1981). The cultures of mesorhizobia and rhizobacterium were maintained on the Yeast Extract Mannitol Agar (YEMA) and the Nutrient Agar (NA) media slants, respectively and stored at $4^{\circ} \mathrm{C}$ for further use during the study period.

\subsection{In-vitro screening of strains of Mesorhizobium sp. for pesticide tolerance}

In this experiment, four recommended pesticides viz. pre-emergence (pendimethalin) and pre-plant (ready-mix which is a combination of pendimethalin + imazethapyr), insecticide (chlorpyrifos) and fungicide (carbendazim) were tested with selected mesorhizobia strains for tolerance to single $(1 x)$ and double $(2 x)$ the recommended doses of pesticides (Table 1$)$ using the disc diffusion method. Sterile filter paper discs were soaked in pesticide solutions in methanol (based on their optimal solubility) at concentrations equivalent to single and double the recommended doses when applied under field conditions (Table 1). Bacterial cultures were grown in YEM broth until they reached the mid-exponential growth phase $(O D 600=0.8)$ before inoculation. Pesticide discs were placed on the minimal salt agar medium plates (Ahemad and Khan 2009) and were uniformly spread with the bacterial cultures. The plates were kept overnight at $4 \circ \mathrm{C}$ to allow diffusion of the pesticide, followed by incubation at $28^{\circ} \mathrm{C}$ for $3-5$ days. Growth of individual Mesorhizobium sp. with different pesticides were compared with control discs dipped in sterile water without pesticides.

Table 1

Concentrations of recommended pesticides at different doses

\begin{tabular}{|c|c|c|c|c|c|}
\hline Pesticides & $\begin{array}{l}\text { Mode of } \\
\text { pesticide } \\
\text { application }\end{array}$ & $\begin{array}{l}\text { Pesticide Dose: } \\
\text { Recommended single doses } \\
((1 x) \text { and double }(2 x) \text { the } \\
\text { recommended doses }\end{array}$ & $\begin{array}{l}\text { Concentration of } \\
\text { recommended } \\
\text { pesticides under } \\
\text { field conditions }\end{array}$ & $\begin{array}{l}\text { Equivalent } \\
\text { concentration } \\
\text { of pesticides in } \\
\text { methanol } \\
(\mu \mathrm{L} \mathrm{ml-1)} \\
{ }^{*}(\mu \mathrm{g} \mathrm{ml}-1)\end{array}$ & $\begin{array}{l}\text { Equivalent } \\
\text { concentration of } \\
\text { pesticides in } \\
\text { Parts/million } \\
\text { (ppm) }\end{array}$ \\
\hline \multirow[t]{2}{*}{$\begin{array}{l}\text { Pendimethalin } 30 \\
\text { EC }\end{array}$} & $\begin{array}{l}\text { pre- } \\
\text { emergence }\end{array}$ & $1 x$ & $0.75 \mathrm{Kg}$ a.i. ha-1 & 6 & $9 \times 104$ \\
\hline & herbicide & $2 x$ & $1.5 \mathrm{Kg}$ a.i. ha-1 & 12 & $18 \times 104$ \\
\hline \multirow{2}{*}{$\begin{array}{l}\text { Ready mix } \\
\text { combination of } \\
\text { imazethpyr }+ \\
\text { pendimethalin } 48 \\
\text { EC }\end{array}$} & \multirow[t]{2}{*}{$\begin{array}{l}\text { pre-plant } \\
\text { herbicide }\end{array}$} & $1 \times$ & $1.2 \mathrm{Kg} \mathrm{Kg}$ a.i. ha-1 & 6 & $2.4 \times 104$ \\
\hline & & $2 x$ & $2.4 \mathrm{Kg}$ a.i. ha-1 & 12 & $4.8 \times 104$ \\
\hline \multirow[t]{2}{*}{ Chlorpyrifos 20 EC } & \multirow{2}{*}{$\begin{array}{l}\text { insecticide } \\
\text { for seed } \\
\text { treatment }\end{array}$} & $1 \times$ & $10 \mathrm{ml}$ a.i. $\mathrm{Kg}-1$ seed & 1.8 & $7.4 \times 104$ \\
\hline & & $2 x$ & $20 \mathrm{ml}$ a.i. $\mathrm{Kg}-1$ seed & 3.6 & $14.8 \times 104$ \\
\hline \multirow[t]{2}{*}{$\begin{array}{l}\text { Carbendazim } 50 \% \\
\text { WP }\end{array}$} & \multirow{2}{*}{$\begin{array}{l}\text { fungicide } \\
\text { for seed } \\
\text { treatment }\end{array}$} & $1 \times$ & $3 \mathrm{~g}$ a.i. $\mathrm{Kg}-1 \mathrm{seed}$ & *3 & $3 \times 103$ \\
\hline & & $2 x$ & $6 \mathrm{~g}$ a.i. $\mathrm{Kg}-1 \mathrm{seed}$ & *6 & $9 \times 103$ \\
\hline
\end{tabular}

Further, the selected bacterial cultures were grown in different pesticides supplemented minimal salt broth media for upto 6 months using sub-culturing technique in $10 \mathrm{~mL}$ tubes at $28 \pm 2^{\circ} \mathrm{C}$ for $48 \mathrm{~h}$ and $130 \mathrm{rpm}$ in a rotatory shaker. Mixed culture of each bacterial strain from four different pesticide amended media were taken in LB medium broth incubated for $48 \mathrm{~h}$ at $28 \pm 2^{\circ} \mathrm{C}$. This technique was conducted to induce possible mutations in bacterial cultures for intrinsic tolerance to pesticides while maintaining stable multifunctional traits. Further experiments were conducted to assess the consistent performance of robust pesticide tolerant strains thus developed invitro, for the multifunctional traits.

\subsection{Effect of different pesticides on multifunctional traits in strains of Mesorhizobium sp. (in-vitro)}

Indole acetic acid (IAA) production of robust pesticide tolerant strains of Mesorhizobium sp. developed in-vitro was determined by the method as demonstrated by Gordon and Weber (1951) later modified by Brick et al. (1991). For this, Luria Bertani (LB) medium broth cultures of the selected pesticide tolerant strains of Mesorhizobium sp. in $(10 \mathrm{ml})$ tubes, each having a fixed concentration of tryptophan $\left(100 \mathrm{gml}^{-1}\right)$ were supplemented with recommended doses of different pesticides (Table 1). It was followed by standard estimation of IAA using orthophosphoric acid and $4 \mathrm{ml}$ of Salkowsky's reagent $\left(2 \% 0.5 \mathrm{M} \mathrm{FeCl}_{3}\right.$ in $35 \%$ perchloric acid). Selected pesticide tolerant 
strains of Mesorhizobium sp. were further tested for siderophore production using the Chrome Azurol S (CAS) agar medium supplemented with recommended doses of different pesticides following the method of Schwyn and Neilands (1987). For hydrogen cyanide (HCN) production, all the pesticide tolerant strains of Mesorhizobium sp. were grown on the HCN induction medium (Bakker and Schipper 1987) amended with the recommended dose of individual pesticides. Cell wall degrading enzyme viz. cellulase and protease production by pesticide tolerant strains of Mesorhizobium sp. was analyzed by the formation of clear zones on the carboxymethylcellulose (CMC) agar and skimmed milk (SM) agar medium, respectively (Vijayabharathi et al. 2018) supplemented with recommended doses of pesticides.

2.4 Compatibility of pesticide tolerant strains of Mesorhizobium sp. and Rhizobacterium RB-1 for functional traits with recommended pesticides

\subsubsection{Seed germination and vigor index (in-vitro)}

Before the field experiment, compatibility of pesticide tolerant strains of Mesorhizobium sp. with rhizobacterium strain RB-1 was tested for growth index using disc plate technique. Lawn of the strain RB-1 was prepared by spreading culture on the NA medium. The sterile discs were dipped in different mesorhizobial cultures and then placed on a lawn of the strain RB-1. The zone of inhibition around the individual disc showed the incompatibility of two cultures. For seed germination experiment, seven treatments were maintained viz. recommended Mesorhizobium cicer strain MR33, Mesorhizobium sp. strain MR2, Mesorhizobium sp. strain MR17, recommended RB-1 + MR33, RB-1 + MR2, RB-1 + MR17 and un-inoculated control (with pesticides application as per recommendation, alone) in Factorial Randomized Block Design (FRBD) with three replications. In co-inoculation treatments, different Mesorhizobium sp. strains along with rhizobacterium strain RB-1 were applied to chickpea seeds in equal ratios (1:1). For seed treatment, healthy seeds of two chickpea varieties viz. PBG7 and GPF2 were surface-sterilized according to Vincet (1970). Surface sterilized seeds were initially treated with fungicide carbendazim (50\% WP @ $3 \mathrm{~g} \mathrm{Kg}^{-1}$ seed) and insecticide chlorpyrifos (20EC @10 ml Kg-1 seed) followed by microbial inoculants as per recommended practice for seed treatment in chickpea. Pendimethalin $\left(9 \times 10^{4} \mathrm{ppm}\right)$ and ready-mix $\left(2.4 \times 10^{4} \mathrm{ppm}\right)$ were incorporated in water agar medium simulating their field application @ $0.75 \mathrm{Kg}$ and $1.2 \mathrm{Kg} \mathrm{ha}^{-1}$, respectively. Treated seeds were allowed to germinate in Petri dish containing $0.7 \%$ water agar with incubation at $28^{\circ} \mathrm{C}$. After 15 days, percent seed germination, fresh weight of seedling, root and shoot length were recorded.

Seedling vigor indices were determined by the following formula:

Seed vigor index I (SVI) $=($ mean of the root length + shoot length $) \times \%$ germination

Seed vigor index II (SVII)=(mean of the fresh weight of root + shoot $) \times$ germination

\subsubsection{Treatment structure for field experiment}

The field investigation was conducted for two consecutive winter seasons (2014-15 and 2015-16) in Factorial Randomized Block Design (FRBD) with three replications. The location of the experiment was at Pulse Research Farm and Microbiology Laboratory, Department of Plant Breeding and Genetics, Punjab Agricultural University, Ludhiana. In total, 42 plots were designed with 4 rows each, having row to row distance of $30 \mathrm{~cm}$, plant to plant distance of $10 \mathrm{~cm}$ and total plot size of $4 \times 2.4=9.6$ sq.m. Chickpea seeds of desi PBG1 and GPF2 varieties were procured from the Pulses Section, Department of Plant Breeding and Genetics, PAU, Ludhiana. The seeds were inoculated with potential pesticide tolerant Mesohizobium sp. strains (MR2 and MR17) and recommended Mesohizobium cicer strain MR33 individually and in combination with the recommended culture of PGP rhizobacterium (RB-1) as per treatment. Monoculture treatment comprised of $20 \mathrm{~g}$ charcoal inoculant per kg of chickpea seeds. For co-inoculation treatments, a cocktail of Mesorhizobium sp. strains and rhizobacterium (RB-1) was made in the ratio of 1:1 for application to chickpea seeds. Before sowing, inoculated seeds were air-dried at room temperature under the shade and sown within two hours. Total 7 microbial inoculant treatments were maintained viz recommended Mesorhizobium cicer sp. strain MR33, Mesorhizobium sp. strain MR2, Mesorhizobium sp. strain MR17, recommended RB$1+$ MR33, RB-1 + MR2, RB-1 + MR17 and un-inoculated control (with recommended pesticides application alone). All the standard agronomic practices were followed for raising the chickpea crop. Chickpea seeds were initially treated with the carbendazim and chlorpyrifos followed by microbial inoculants (Table 1). Readymix and pendimethalin were applied as pre-plant and pre-emergence herbicides (Table 1), respectively in the plots to control the broad-leaved weeds according to recommended management practices.

\subsubsection{Plant growth parameters}


Emergence count was recorded at days after sowing (10 DAS) from central rows of each plot after leaving two border rows on each side and calculated from number of emerged seedlings per meter row length. Observations for plant height, dry weight of roots and shoots, chlorophyll and carotenoid contents were taken after 60 and 90 DAS. For measuring plant height, three plants were randomly selected from each plot and uprooted. Roots were removed from shoots and the height of shoots was measured from the base in $\mathrm{cm}$. Similarly for dry weight of shoots, three randomly selected plants were uprooted from each plot. Roots were detached from shoots, sun dried and then oven dried at $60^{\circ} \mathrm{C}$ for 2 days. Dry weight of shoot and root was recorded in $\mathrm{g}$. Chlorophyll and carotenoid contents were determined as per the methods of Witham et al. (1971) and Dere et al. (1998), respectively.

\subsubsection{Antioxidant enzymes activities in root exudates}

For collection of root exudates, frozen root and shoot tissues of each treatment were homogenized at $4^{\circ} \mathrm{C}$ in an ice-chilled mortar in QB buffer per gram of tissue (Kumar et al. 2009). Crude homogenates were centrifuged at $15000 \mathrm{~g}$ for $15 \mathrm{~min}$ at $4{ }^{\circ} \mathrm{C}$, and the supernatant fractions were frozen at $-20^{\circ} \mathrm{C}$. Superoxide dismutase (SOD) activity and catalase (CAT) activity were recorded at 120 DAS by the methods of Aebi (1983) and Marklund and Marklund (1974), respectively.

\subsubsection{Symbiotic nitrogen fixation}

Observations of nodule number, dry weight of nodules and leghaemoglobin content were recorded at 60 and 90 DAS. For evaluation of symbiotic parameters, three randomly selected plants were uprooted from each plot with their root system being intact. The roots were washed in running tap water and nodules carefully detached with forceps. The number of nodules per plant was recorded by taking average. The detached nodules were oven dried at $60^{\circ} \mathrm{C}$ for 2 days and the dry weight of nodules per plant was recorded in $\mathrm{mg}$. Leghaemoglobin content was estimated according to Wilson and Reisenauer (1963) by reading absorbance of clear nodular tissue extract using Drabkin's solution at $540 \mathrm{~nm}$.

\subsubsection{Soil health}

Soil enzyme activities were analyzed at 90 DAS. Estimation of Dehydrogenase activity of soil was based on production of Triphenyl formazan (TPF) from Triphenyl Tetrazolium Chloride (Klosse and Tabatabai 2000). Soil Urease activity was activity was calculated as $\mu \mathrm{g}$ of ammonium $\mathrm{N}$ released $\mathrm{g}^{-1}$ of soil $2 \mathrm{hr}^{-1}$ (Tabatabai 1982).

\subsubsection{Yield attributing traits, seed protein content and grain yield}

Yield attributing traits, Seed protein content and Grain yield were determined at harvesting stage. The number of pods per plant was recorded by taking average of three plants were randomly selected at harvesting stage. The number of seeds per pod was recorded by taking average of seeds from three randomly selected plants at harvesting stage. Crop was sickle harvested and grains were thrashed. Hundred-seed weight was measured on a per-plot basis by taking a sample of the harvested seed in each plot. Total protein content of seeds was determined by Kjeldahl's technique with slight modification (McKenzie and Wallace 1954).

\subsection{Statistical analysis}

Data were analyzed using Analysis of Variance (ANOVA) based on the pooled mean of two years with SAS Statistical Package Version 9.3. Further, mean separation of treatment effects was accomplished using the Tukey test. The least significant difference (LSD) was calculated at the $5 \%$ probability level.

\section{Results}

\subsection{Tolerance of strains of Mesorhizobium sp. with recommended pesticides (in-vitro)}

Confirmatory tests based on Hoffer's alkaline medium and ketolactose tests showed white translucent colonies in 50 strains of Rhizobium where as red colonies indicated Agrobacterium. as contaminant. All the rhizobia showed growth at $28^{\circ} \mathrm{C}$ on YEMA medium after 48-72 hrs and considered as Mesorhizobium. All the strains of Mesorhizobium sp. (50) were further subjected to screening for pesticide tolerance by the disc diffusion method. The growth inhibition zones around pesticide discs allowed us to divide the bacterial strains under study into three categories viz. luxuriant, moderate and poor tolerant. The highest growth index was mainly expressed in terms of percentage (\%) of luxuriantly tolerant strains, whereas the lowest growth index was expressed in terms of poorly tolerant strains. Among different pesticide treatments at recommended doses, higher growth index was observed with pendimethalin (37\%) as compared to ready-mix (36\%), chlorpyrifos (31\%) and carbendazim (30\%) (Plate 1 and Fig. 1). An overall decline in growth index was 
noticed with $2 \times$ recommended doses of all the pesticides. viz. overall $8 \%$ decline in luxuriantly tolerant strains with $2 \times$ pendimethalin and $2 \times$ ready-mix each, followed by $5 \%$ with $2 \times$ chlorpyrifos and $10 \%$ with $2 \times$ carbendazim. However, the maximum decline in growth index was witnessed for chlorpyrifos Based on this experiment, 20 strains of Mesorhizobium sp. were found to have the potential to be selected as pesticide tolerant strains. This test showed that among different pesticides, the highest inhibitory effect on the growth of Mesorhizobium strains was exhibited by insecticide (chlorpyrifos) followed by fungicide (carbendazim) while both the herbicides (pendimethalin and ready-mix of pendimethalin + imazthpyr) were comparatively less toxic to the growth of selected Mesorhizobium strains.

\subsection{Effect of recommended pesticides on multifunctional traits in strains Mesorhizobium sp.}

The selected robust strains of Mesorhizobium sp. with intrinsic tolerance to different pesticides developed over several generations invitro were further analyzed for multifunctional traits viz. IAA, siderophore and HCN production with recommended pesticide treatments. For IAA production, the selected strains of Mesorhizobium sp., differed significantly with all the pesticides in presence (Trp+) and absence (Trp-) of tryptophan (Figs. 2a-d). Additionally, IAA production significantly increased with Trp + over Trp- in different treatments. Among different pesticide treatments, significantly high IAA production was observed for Mesorhizobium sp. strain MR2 $\left(48.5 \mu \mathrm{gml}^{-1}\right)$ with pendimethalin (Plate 2) followed by ready-mix, carbendazim and chlorpyrifos (39.8, 25.2 and $23.2 \mu \mathrm{g} \mathrm{ml}^{-1}$, respectively). However, an overall decline in IAA production by 1.2 fold was recorded with ready-mix followed by carbendazim ( 1.6 fold) and chlorpyrifos (2.3 fold) over the control. Further, siderophore production by the Mesorhizobium strains with recommended pesticides was shown by the appearance of clear zones around the bacterial colonies due to the chelation of iron bound to CAS dye (Plate 3a-c). Among 20 pesticide tolerant Mesorhizobium sp., only the strains MR2, MR17 and recommended MR33 were tested positive for siderophore production on CAS medium amended with recommended doses of pendimethalin and ready-mix treatments, whereas, with chlorpyrifos only strain MR2 produced siderophores. However, carbendazim amended CAS agar medium was inhibitory for siderophore production by Mesorhizobium sp. strains MR2, MR17 and recommended strain MR33. The remaining strains of Mesorhizobium sp. along with Mesorhizobium sp. strains MR2, MR17 and recommended MR33 appeared negative for siderophore production with the pesticides (pendimethalin, ready-mix and chlorpyrifos). Further for cell wall degrading enzyme production, among all the pesticide tolerant strains, only Mesorhizobium sp. strain MR17 was positive for protease production in presence of pendimethalin and ready-mix while it presented cellulase production with only pendimethalin amended SM agar medium (Plates 4a-c). However, there was an absence of any cell wall degrading enzyme production in the remaining Mesorhizobium sp. strains. Based on the in-vitro evaluation of multifunctional traits, it was observed that insecticide (chlorpyrifos) exhibited a highest inhibitory effect on metabolism of Mesorhizobium sp. strains followed by fungicide (carbendazim) while both the herbicides (pendimethalin and ready-mix of pendimethalin + imazthpyr) were less toxic towards selected Mesorhizobium strains, accounting for a comparatively better exhibition of multifunctional traits.

\subsection{Compatibility of pesticide tolerant strains of Mesorhizobium sp. and Rhizobacterium RB-1 for functional traits with recommended} pesticides

\subsubsection{Seed germination and vigor index (in-vitro)}

Based on multifunctional traits, two strains of Mesorhizobium sp. viz. MR2, MR17 along with recommended strain MR33 were selected as potential pesticide tolerant strains. In-vitro compatibility test of individual potential pesticide tolerant strains of Mesorhizobium sp. with the recommended culture of RB-1 revealed that all the dual-inoculants were compatible (Plates 5a-g). The effects of dual-inoculant treatments on seed germination in chickpea were significantly different. The highest percentage seed germination (92\%), radical length $(4.3 \mathrm{~cm})$, plumule length $(9.8 \mathrm{~cm})$, fresh weight of seedling $(0.49 \mathrm{~g})$, vigor index I $(1367.1)$ and vigor index II $(44.9)$ were found with Mesorhizobium sp. strain MR2 as compared to un-inoculated control treatment (89\%, 4.3cm, $7.0 \mathrm{~cm}, 0.39 \mathrm{~g}, 1012.0,34.8$, respectively). Dual inoculation of MR2 with RB1 further increased the seed germination (1.1 fold), radical length ( 1.9 fold), plumule length ( 1.6 fold), fresh weight of seedling (1.4 fold), vigor index I (1.8 fold) and vigor index II (1.5 fold) over un-inoculated control treatment (Tables 2 and 3). However, PBG7 and GPF2 chickpea varieties were at par for seedling germination. All the potential Mesorhizobium strains under study significantly improved the vigor index (1.2-1.3 fold) and germination (2.2-3.4\%) which further increased by $1.7-1.8$ fold and by 3.4-6.7\%, respectively, with individual dual-inoculant treatments over un-inoculated control. However, RB-1 + MR2 performed at par with recommended RB-1 + MR33 for enhancement of seed germination and fresh weight of seedling and vigor index II, whereas, for an increase in plumule length RB-1 + MR17 was at par with recommended RB-1 + MR33. This experiment further confirmed the potential of selected pesticide tolerant strain of Mesorhizobium sp. viz. MR2 and MR17 along with recommended strain MR33 for enhancement of growth and yield in chickpea. However, the ecological performance of all the selected Mesorhizobium strains alone and individual dual

Page 6/24 
inoculation treatments with RB-1 was assessed under field conditions for a better understanding of chickpea-Mesorhizobium symbiosis under pesticide stressed field conditions.

Table 2

Effect of potential pesticide tolerant strains of Mesorhizobium sp. and rhizobacterium RB-1 on seedling germination

\begin{tabular}{|c|c|c|c|c|c|c|c|c|c|c|c|c|}
\hline \multirow[t]{2}{*}{ Treatments } & \multicolumn{3}{|c|}{ Seed germination (\%) } & \multicolumn{3}{|c|}{ Plumule length (cm) } & \multicolumn{3}{|c|}{ Radical length (cm) } & \multicolumn{3}{|c|}{$\begin{array}{l}\text { Fresh weight of seedling } \\
\text { (g) }\end{array}$} \\
\hline & PBG7 & GPF2 & Mean & PBG7 & GPF2 & Mean & PBG7 & GPF2 & Mean & PBG7 & GPF2 & Mean \\
\hline Control & $\begin{array}{l}90 \pm \\
1.76\end{array}$ & $\begin{array}{l}88 \pm \\
2.08\end{array}$ & $\begin{array}{l}89 \pm \\
1.28 \mathrm{a}\end{array}$ & $\begin{array}{l}7.3 \pm \\
0.27\end{array}$ & $\begin{array}{l}6.8 \pm \\
0.26\end{array}$ & $\begin{array}{l}7.0 \pm \\
0.20 \mathrm{a}\end{array}$ & $\begin{array}{l}4.5 \pm \\
0.26\end{array}$ & $\begin{array}{l}4.2 \pm \\
0.23\end{array}$ & $\begin{array}{l}4.3 \pm \\
0.17 a\end{array}$ & $\begin{array}{l}0.41 \\
\pm \\
0.02\end{array}$ & $\begin{array}{l}0.37 \\
\pm \\
0.03\end{array}$ & $\begin{array}{l}0.39 \pm \\
0.02 a\end{array}$ \\
\hline MR33 & $\begin{array}{l}92 \pm \\
1.20\end{array}$ & $\begin{array}{l}91 \pm \\
1.73\end{array}$ & $\begin{array}{l}91 \pm \\
0.95 f\end{array}$ & $\begin{array}{l}9.3 \pm \\
0.29\end{array}$ & $\begin{array}{l}8.7 \pm \\
0.29\end{array}$ & $\begin{array}{l}9.0 \pm \\
0.23 f\end{array}$ & $\begin{array}{l}5.2 \pm \\
0.27\end{array}$ & $\begin{array}{l}4.4 \pm \\
0.23\end{array}$ & $\begin{array}{l}4.8 \pm \\
0.24 f\end{array}$ & $\begin{array}{l}0.46 \\
\pm \\
0.03\end{array}$ & $\begin{array}{l}0.43 \\
\pm \\
0.03\end{array}$ & $\begin{array}{l}0.44 \pm \\
0.02 \mathrm{abc}\end{array}$ \\
\hline MR2 & $\begin{array}{l}93 \pm \\
1.76\end{array}$ & $\begin{array}{l}92 \pm \\
0.88\end{array}$ & $\begin{array}{l}92 \pm \\
0.91 \mathrm{e}\end{array}$ & $\begin{array}{l}9.6 \pm \\
0.35\end{array}$ & $\begin{array}{l}9.9 \pm \\
0.32\end{array}$ & $\begin{array}{l}9.8 \pm \\
0.22 \mathrm{e}\end{array}$ & $\begin{array}{l}5.2 \pm \\
0.23\end{array}$ & $\begin{array}{l}4.9 \pm \\
0.29\end{array}$ & $\begin{array}{l}5.1 \pm \\
0.18 \mathrm{e}\end{array}$ & $\begin{array}{l}0.51 \\
\pm \\
0.03\end{array}$ & $\begin{array}{l}0.46 \\
\pm \\
0.03\end{array}$ & $\begin{array}{l}0.49 \pm \\
0.02 \mathrm{abc}\end{array}$ \\
\hline MR17 & $\begin{array}{l}91 \pm \\
1.53\end{array}$ & $\begin{array}{l}91 \pm \\
1.67\end{array}$ & $\begin{array}{l}91 \pm \\
1.01 \mathrm{fg}\end{array}$ & $\begin{array}{l}9.2 \pm \\
0.35\end{array}$ & $\begin{array}{l}8.4 \pm \\
0.32\end{array}$ & $\begin{array}{l}8.8 \pm \\
0.29 \mathrm{~g}\end{array}$ & $\begin{array}{l}5.1 \pm \\
0.26\end{array}$ & $\begin{array}{l}4.7 \pm \\
0.37\end{array}$ & $\begin{array}{l}4.9 \pm \\
0.22 f g\end{array}$ & $\begin{array}{l}0.47 \\
\pm \\
0.03\end{array}$ & $\begin{array}{l}0.42 \\
\pm \\
0.03\end{array}$ & $\begin{array}{l}0.44 \pm \\
0.02 \mathrm{abc}\end{array}$ \\
\hline $\begin{array}{l}\text { RB-1 + MR33 } \\
\text { (Recommended) }\end{array}$ & $\begin{array}{l}96 \pm \\
0.88\end{array}$ & $\begin{array}{l}95 \pm \\
0.67\end{array}$ & $\begin{array}{l}95 \pm \\
0.54 b\end{array}$ & $\begin{array}{l}11.1 \\
\pm \\
0.32\end{array}$ & $\begin{array}{l}10.6 \\
\pm \\
0.35\end{array}$ & $\begin{array}{l}10.9 \pm \\
0.24 c\end{array}$ & $\begin{array}{l}7.9 \pm \\
0.29\end{array}$ & $\begin{array}{l}7.4 \pm \\
0.19\end{array}$ & $\begin{array}{l}7.7 \pm \\
0.19 d\end{array}$ & $\begin{array}{l}0.55 \\
\pm \\
0.02\end{array}$ & $\begin{array}{l}0.54 \\
\pm \\
0.03\end{array}$ & $\begin{array}{l}0.55 \pm \\
0.02 \mathrm{bc}\end{array}$ \\
\hline RB-1 + MR2 & $\begin{array}{l}95 \pm \\
1.45\end{array}$ & $\begin{array}{l}94 \pm \\
0.58\end{array}$ & $\begin{array}{l}94 \pm \\
0.71 \mathrm{bc}\end{array}$ & $\begin{array}{l}11.5 \\
\pm \\
0.44\end{array}$ & $\begin{array}{l}10.8 \\
\pm \\
0.35\end{array}$ & $\begin{array}{l}11.2 \pm \\
0.29 b\end{array}$ & $\begin{array}{l}8.4 \pm \\
0.26\end{array}$ & $\begin{array}{l}8.0 \pm \\
0.17\end{array}$ & $\begin{array}{l}8.2 \pm \\
0.17 b\end{array}$ & $\begin{array}{l}0.58 \\
\pm \\
0.03\end{array}$ & $\begin{array}{l}0.54 \\
\pm \\
0.03\end{array}$ & $\begin{array}{l}0.56 \pm \\
0.02 b\end{array}$ \\
\hline RB-1 + MR17 & $\begin{array}{l}93 \pm \\
1.45\end{array}$ & $\begin{array}{l}92 \pm \\
1.15\end{array}$ & $\begin{array}{l}92 \pm \\
0.84 d\end{array}$ & $\begin{array}{l}11.1 \\
\pm \\
0.44\end{array}$ & $\begin{array}{l}10.4 \\
\pm \\
0.39\end{array}$ & $\begin{array}{l}10.8 \pm \\
0.28 \mathrm{~cd}\end{array}$ & $\begin{array}{l}8.1 \pm \\
0.21\end{array}$ & $\begin{array}{l}7.9 \pm \\
0.17\end{array}$ & $\begin{array}{l}8.0 \pm \\
0.17 \mathrm{c}\end{array}$ & $\begin{array}{l}0.53 \\
\pm \\
0.04\end{array}$ & $\begin{array}{l}0.47 \\
\pm \\
0.04\end{array}$ & $\begin{array}{l}0.50 \pm \\
0.03 \mathrm{abc}\end{array}$ \\
\hline Mean & $\begin{array}{l}93 \pm \\
1.43\end{array}$ & $\begin{array}{l}92 \pm \\
1.25\end{array}$ & & $\begin{array}{l}9.9 \pm \\
0.3\end{array}$ & $\begin{array}{l}9.4 \pm \\
0.3\end{array}$ & & $\begin{array}{l}6.3 \pm \\
0.26\end{array}$ & $\begin{array}{l}5.9 \pm \\
0.26\end{array}$ & & $\begin{array}{l}0.50 \\
\pm \\
0.03\end{array}$ & $\begin{array}{l}0.47 \\
\pm \\
0.04\end{array}$ & \\
\hline Variety (V) & 0.60 & & & 0.74 & & & 0.82 & & & 0.73 & & \\
\hline Treatment (T) & 1.1 & & & 0.14 & & & 0.15 & & & 0.14 & & \\
\hline$V \times T$ & NS & & & 0.19 & & & 0.22 & & & NS & & \\
\hline
\end{tabular}


Table 3

Effect of potential pesticide tolerant strains of Mesorhizobium sp. and rhizobacterium RB-1 on vigour index

\begin{tabular}{|c|c|c|c|c|c|c|}
\hline \multirow[t]{2}{*}{ Treatments } & \multicolumn{3}{|l|}{ Vigor Index I } & \multicolumn{3}{|l|}{ Vigor Index II } \\
\hline & PBG7 & GPF2 & Mean & PBG7 & GPF2 & Mean \\
\hline CONTROL & $\begin{array}{l}1053.9 \pm \\
68.06\end{array}$ & $970.0 \pm 66.65$ & $1012.0 \pm 46.55 a$ & $36.8 \pm 2.61$ & $32.7 \pm 3.63$ & $34.8 \pm 2.21 a$ \\
\hline MR33 & $\begin{array}{l}1330.5 \pm \\
68.01\end{array}$ & $\begin{array}{l}1193.9 \pm \\
69.98\end{array}$ & $1262.2 \pm 53.26 f$ & $42.2 \pm 3.00$ & $38.9 \pm 3.73$ & $40.6 \pm 2.26 f$ \\
\hline MR2 & $\begin{array}{l}1376.5 \pm \\
79.38\end{array}$ & $\begin{array}{l}1357.7 \pm \\
68.55\end{array}$ & $1367.1 \pm 47.09 \mathrm{e}$ & $47.4 \pm 3.38$ & $42.5 \pm 3.31$ & $44.9 \pm 2.37 e$ \\
\hline MR17 & $\begin{array}{l}1303.0 \pm \\
76.12\end{array}$ & $\begin{array}{l}1189.4 \pm \\
79.46\end{array}$ & $1246.2 \pm 55.38 f$ & $42.6 \pm 3.58$ & $38.2 \pm 3.62$ & $40.4 \pm 2.48 \mathrm{fg}$ \\
\hline $\begin{array}{l}\text { RB-1 + LGR33 } \\
\text { (Recommended) }\end{array}$ & $\begin{array}{l}1821.9 \pm \\
74.40\end{array}$ & $\begin{array}{l}1710.9 \pm \\
60.56\end{array}$ & $1766.4 \pm 49.56 d$ & $53.0 \pm 2.75$ & $51.5 \pm 3.58$ & $52.2 \pm 2.05 b c$ \\
\hline $\mathrm{RB}-1+\mathrm{MR} 2$ & $\begin{array}{l}1885.9 \pm \\
94.97\end{array}$ & $\begin{array}{l}1774.1 \pm \\
62.45\end{array}$ & $1830.0 \pm 56.59 \mathrm{~b}$ & $55.3 \pm 3.30$ & $50.5 \pm 3.40$ & $52.9 \pm 2.38 b$ \\
\hline RB-1 + MR17 & $\begin{array}{l}1781.1 \pm \\
87.09\end{array}$ & $\begin{array}{l}1684.9 \pm \\
72.62\end{array}$ & $1733 \pm 55.8 \mathrm{~g}$ & $48.9 \pm 4.51$ & $43.6 \pm 4.32$ & $46.3 \pm 3.03 d$ \\
\hline Mean & $1508 \pm 78.3^{*}$ & $1412 \pm 68.6$ & & $46.6 \pm 3.30^{*}$ & $42.6 \pm 3.66$ & \\
\hline Variety (V) & 13.9 & & & 0.68 & & \\
\hline Treatment $(\mathrm{T})$ & 26.1 & & & 1.3 & & \\
\hline$V \times T$ & 36.8 & & & NS & & \\
\hline
\end{tabular}

\subsubsection{Plant growth promotion}

Seedling's emergence count has been expressed as percentage (\%) germination of seeds. This revealed a significant variation among all the microbial inoculant treatments in respect of the un-inoculated control. With the mono-inoculant treatments, seed germination significantly enhanced, ranged from 5.6-8.7\% over the un-inoculated control. The highest germination was recorded with recommended Mesorhizobium cicer strain MR33 (92\%) followed by Mesorhizobium sp. strain MR2 (91\%) and Mesorhizobium sp. strain MR17 (89\%) as compared to the un-inoculated control (84\%). The germination significantly enhanced with dual-inoculant treatment of recommended RB-1 + MR33 (4.3\%) followed by RB-1 + MR2 (3.3\%) over MR33 and MR2 alone treatments, respectively. However, recommended RB-1 + MR33 (96\%) performed at par with RB-1 + MR2 (94\%), whereas RB-1 + MR17 resulted in the minimum germination (92\%). The pooled mean of plant height revealed that the PBG7 variety $(2.2 \%)$ was significantly better over the GPF2 chickpea variety. Significant interaction was not observed between the varieties and treatments on emergence count.

For enhancement of plant height at $60 \mathrm{DAS}$, all the mono-inoculant treatments varied significantly by $10.2-19.1 \%$ over the un-inoculated control (Table 4). The effect of dual-inoculant treatments on plant height further increased by $25.0-33.9 \%$ over the control. In similarity with emergence count, dual-inoculant treatments of pesticide tolerant Mesorhizobium sp. strains with rhizobacterium RB-1 significantly improved the plant height over Mesorhizobium alone treatments. The highest improvement was obtained with recommended RB-1 + MR33 (13.6\%) over MR33 alone followed by RB-1 + MR2 (7.8\%) over MR2 alone treatment. Further, PBG7 variety performed significantly better by $10.1 \%$ over GPF2 chickpea variety for enhancement of the plant height. A similar trend was followed at 90 DAS, where there was significant variation in plant height from $8.9-17.1 \%$ with the mono-inoculant treatments over un-inoculated control. All the dualinoculant treatments, further increased the plant height ranged from 16.5-22.7\% over un-inoculated control, however, RB-1 + MR2 was at par with RB-1 + MR17. Recommended RB-1 + MR33 presented the most abundant increase in plant height (11.0\%) over MR33 alone treatment. Moreover, plant height significantly increased with PBG7 variety over GPF2 variety by $10.5 \%$. The interaction between variety and treatment was not significant at 60 and 90 DAS, respectively. 
Table 4

Effect of potential pesticide tolerant strains of Mesorhizobium sp. and rhizobacterium RB-1 on plant growth

\begin{tabular}{|c|c|c|c|c|c|c|c|c|c|}
\hline \multirow[t]{3}{*}{ Treatments } & \multirow{2}{*}{\multicolumn{3}{|c|}{$\begin{array}{l}\text { Plant Height (cm plant }{ }^{-1} \text { ) } \\
60 \text { DAS (90 DAS) }\end{array}$}} & \multirow{2}{*}{\multicolumn{3}{|c|}{$\begin{array}{l}\text { Dry weight of shoot (g plant }{ }^{-1} \text { ) } \\
60 \text { DAS ( } 90 \text { DAS) }\end{array}$}} & \multirow{2}{*}{\multicolumn{3}{|c|}{$\begin{array}{l}\text { Dry weight of root }\left(\text { g plant }^{-1} \text { ) }\right. \\
60 \text { DAS (90 DAS) }\end{array}$}} \\
\hline & & & & & & & & & \\
\hline & PBG7 & GPF2 & $\begin{array}{l}\text { Overall } \\
\text { Mean }\end{array}$ & PBG7 & GPF2 & $\begin{array}{l}\text { Overall } \\
\text { Mean }\end{array}$ & PBG7 & GPF2 & $\begin{array}{l}\text { Overall } \\
\text { Mean }\end{array}$ \\
\hline \multirow[t]{2}{*}{ Control } & $\begin{array}{l}33.4 \\
\pm 1.81\end{array}$ & \multirow{2}{*}{$\begin{array}{l}31.4 \\
\pm \\
1.27 \\
(45.2 \\
\pm \\
1.42)\end{array}$} & $\begin{array}{l}32.4 \pm \\
1.09 \mathrm{ac}\end{array}$ & $\begin{array}{l}2.23 \pm \\
0.18\end{array}$ & \multirow{2}{*}{$\begin{array}{l}2.04 \pm \\
0.17 \\
(2.84 \pm \\
0.24)\end{array}$} & \multirow[t]{2}{*}{$\begin{array}{l}2.14 \pm \\
0.12 \mathrm{a}(3.20 \\
\pm 0.25 \mathrm{a})\end{array}$} & \multirow{2}{*}{$\begin{array}{l}0.94 \pm \\
0.09 \\
(1.96 \pm \\
0.17)\end{array}$} & \multirow{2}{*}{$\begin{array}{l}0.83 \pm \\
0.06 \\
(1.71 \pm \\
0.12)\end{array}$} & \multirow[t]{2}{*}{$\begin{array}{l}0.89 \pm 0.04 a \\
(1.84 \pm \\
0.11 a)\end{array}$} \\
\hline & $\begin{array}{l}(48.3 \\
\pm \\
0.78)\end{array}$ & & $\begin{array}{l}(46.7 \pm \\
0.90 \mathrm{a})\end{array}$ & $\begin{array}{l}(3.57 \pm \\
0.41)\end{array}$ & & & & & \\
\hline \multirow[t]{2}{*}{ MR33 } & $\begin{array}{l}41.4 \\
\pm 1.29\end{array}$ & \multirow{2}{*}{$\begin{array}{l}35.0 \\
\pm \\
2.10 \\
(48.1 \\
\pm \\
0.99)\end{array}$} & $\begin{array}{l}38.2 \pm \\
1.54 \mathrm{e}\end{array}$ & $\begin{array}{l}2.70 \pm \\
0.16\end{array}$ & \multirow{2}{*}{$\begin{array}{l}2.22 \pm \\
0.19 \\
(3.25 \pm \\
0.19)\end{array}$} & \multirow{2}{*}{$\begin{array}{l}2.46 \pm 0.14 \mathrm{e} \\
(3.73 \pm \\
0.24 \mathrm{e})\end{array}$} & \multirow{2}{*}{$\begin{array}{l}1.07 \pm \\
0.10 \\
(2.10 \pm \\
0.15)\end{array}$} & \multirow{2}{*}{$\begin{array}{l}0.92 \pm \\
0.06 \\
(1.87 \pm \\
0.16)\end{array}$} & \multirow[t]{2}{*}{$\begin{array}{l}0.99 \pm 0.05 a \\
(1.98 \pm \\
0.11 e)\end{array}$} \\
\hline & $\begin{array}{l}(55.1 \\
\pm \\
1.40)\end{array}$ & & $\begin{array}{l}(51.6 \pm \\
1.34 f)\end{array}$ & $\begin{array}{l}(4.21 \pm \\
0.34)\end{array}$ & & & & & \\
\hline \multirow[t]{2}{*}{ MR2 } & $\begin{array}{l}40.8 \\
\pm 1.23\end{array}$ & \multirow{2}{*}{$\begin{array}{l}36.4 \\
\pm \\
1.58 \\
(51.6 \\
\pm \\
1.36)\end{array}$} & $\begin{array}{l}38.6 \pm \\
1.16 \mathrm{ef}\end{array}$ & $\begin{array}{l}2.75 \pm \\
0.16\end{array}$ & \multirow{2}{*}{$\begin{array}{l}2.13 \pm \\
0.23 \\
(3.30 \pm \\
0.19)\end{array}$} & \multirow{2}{*}{$\begin{array}{l}2.44 \pm \\
0.16 \mathrm{ef} \\
3(.79 \pm \\
0.24 \mathrm{ef})\end{array}$} & \multirow{2}{*}{$\begin{array}{l}1.03 \pm \\
0.09 \\
(2.10 \pm \\
0.16)\end{array}$} & \multirow{2}{*}{$\begin{array}{l}0.87 \pm \\
0.07 \\
(1.90 \pm \\
0.16)\end{array}$} & \multirow[t]{2}{*}{$\begin{array}{l}0.95 \pm 0.05 a \\
(2.00 \pm \\
0.11 \mathrm{ef})\end{array}$} \\
\hline & $\begin{array}{l}(57.8 \\
\pm \\
1.45)\end{array}$ & & $\begin{array}{l}(54.7 \pm \\
1.34 c d e)\end{array}$ & $\begin{array}{l}(4.28 \pm \\
0.35)\end{array}$ & & & & & \\
\hline \multirow[t]{2}{*}{ MR17 } & $\begin{array}{l}37.9 \\
\pm 2.20\end{array}$ & \multirow{2}{*}{$\begin{array}{l}33.5 \\
\pm \\
1.56 \\
(46.3 \\
\pm \\
2.03)\end{array}$} & $\begin{array}{l}35.7 \pm \\
1.46 \mathrm{~g}\end{array}$ & \multirow{2}{*}{$\begin{array}{l}2.34 \pm \\
0.16 \\
(3.75 \pm \\
0.40)\end{array}$} & \multirow{2}{*}{$\begin{array}{l}1.98 \pm \\
0.18 \\
(2.93 \pm \\
0.26)\end{array}$} & $\begin{array}{l}2.16 \pm \\
0.13 a\end{array}$ & \multirow{2}{*}{$\begin{array}{l}0.95 \pm \\
0.09 \\
(2.01 \pm \\
0.15)\end{array}$} & \multirow{2}{*}{$\begin{array}{l}0.84 \pm \\
0.06 \\
(1.77 \pm \\
0.14)\end{array}$} & \multirow[t]{2}{*}{$\begin{array}{l}0.90 \pm 0.04 a \\
(1.89 \pm \\
0.11 a)\end{array}$} \\
\hline & $\begin{array}{l}(55.6 \\
\pm \\
1.33)\end{array}$ & & $\begin{array}{l}(50.9 \pm \\
1.81 \mathrm{~g})\end{array}$ & & & $\begin{array}{l}(3.34 \pm \\
0.26 a)\end{array}$ & & & \\
\hline \multirow[t]{2}{*}{$\begin{array}{l}\text { RB-1 + MR33 } \\
\text { (Recommended) }\end{array}$} & $\begin{array}{l}45.3 \\
\pm 2.09\end{array}$ & \multirow{2}{*}{$\begin{array}{l}41.4 \\
\pm \\
1.48 \\
(56.4 \\
\pm \\
2.42)\end{array}$} & $\begin{array}{l}43.4 \pm \\
1.30 \mathrm{~b}\end{array}$ & \multirow{2}{*}{$\begin{array}{l}3.16 \pm \\
0.18 \\
(4.49 \pm \\
0.41)\end{array}$} & $\begin{array}{l}2.52 \pm \\
0.21 \\
(394+\end{array}$ & $\begin{array}{l}2.84 \pm \\
0.16 \mathrm{~b}\end{array}$ & $\begin{array}{l}1.28 \pm \\
0.13 \\
0.47+\end{array}$ & $\begin{array}{l}1.08 \pm \\
0.11 \\
0.07+\end{array}$ & $\begin{array}{l}1.18 \pm 0.07 a \\
(2.27 \pm\end{array}$ \\
\hline & $\begin{array}{l}(58.3 \\
\pm \\
0.72)\end{array}$ & & $\begin{array}{l}(57.3 \pm \\
1.24 b)\end{array}$ & & $0.33)$ & $\begin{array}{l}(4.21 \pm \\
0.26 \mathrm{bc})\end{array}$ & $0.09)$ & $0.12)$ & \\
\hline RB-1 + MR2 & $\begin{array}{l}43.2 \\
\pm 1.60\end{array}$ & $\begin{array}{l}40.0 \\
\pm \\
1.21\end{array}$ & $\begin{array}{l}41.6 \pm \\
1.09 c\end{array}$ & $\begin{array}{l}3.16 \pm \\
0.21 \\
(4.56 \pm\end{array}$ & $\begin{array}{l}2.59 \pm \\
0.22 \\
(4.02 \pm\end{array}$ & $\begin{array}{l}2.88 \pm \\
0.17 \mathrm{bc}\end{array}$ & $\begin{array}{l}1.23 \pm \\
0.14 \\
(2.46 \pm\end{array}$ & $\begin{array}{l}1.02 \pm \\
0.10 \\
(2.13 \pm\end{array}$ & $\begin{array}{l}1.13 \pm 0.07 a \\
(2.29 \pm \\
0.09 b)\end{array}$ \\
\hline & $\begin{array}{l}(57.1 \\
\pm \\
0.75)\end{array}$ & $\begin{array}{l}(52.0 \\
\pm \\
2.05)\end{array}$ & $\begin{array}{l}(54.5 \pm \\
1.35 c)\end{array}$ & 0.41) & $0.32)$ & $\begin{array}{l}(4.29 \pm \\
0.26 b)\end{array}$ & $0.11)$ & $0.13)$ & \\
\hline RB-1 + MR17 & $\begin{array}{l}42.4 \\
\pm 2.02\end{array}$ & $\begin{array}{l}38.6 \\
\pm \\
143\end{array}$ & $\begin{array}{l}40.5 \pm \\
1.38 \mathrm{~cd}\end{array}$ & $\begin{array}{l}3.06 \pm \\
021(4.40 \\
+0.38)\end{array}$ & $\begin{array}{l}2.31 \pm \\
0.19\end{array}$ & $\begin{array}{l}2.69 \pm \\
0.18 \mathrm{bcd}\end{array}$ & $\begin{array}{l}1.14 \pm \\
0.12 \\
(2.35 \pm\end{array}$ & $\begin{array}{l}0.95 \pm \\
0.10 \\
1.96+\end{array}$ & $\begin{array}{l}1.05 \pm 0.06 \mathrm{a} \\
(2.16 \pm \\
0.10 \mathrm{bcd})\end{array}$ \\
\hline & $\begin{array}{l}(56.5 \\
\pm \\
1.61)\end{array}$ & $\begin{array}{l}(52.4 \\
\pm \\
1.77)\end{array}$ & $\begin{array}{l}(54.4 \pm \\
1.30 \mathrm{~cd})\end{array}$ & & $\begin{array}{l}(3.90 \pm \\
0.31)\end{array}$ & $\begin{array}{l}(4.15 \pm \\
0.25 b c d)\end{array}$ & $0.13)$ & $0.10)$ & \\
\hline Mean & $\begin{array}{l}40.7 \\
\pm \\
1.40^{\star}\end{array}$ & $\begin{array}{l}36.6 \\
\pm \\
1.34\end{array}$ & & $\begin{array}{l}2.77 \pm \\
0.14 \\
(4.18 \pm\end{array}$ & $\begin{array}{l}2.26 \pm \\
0.088\end{array}$ & & $\begin{array}{l}1.09 \pm \\
0.05 \\
(2.21 \pm\end{array}$ & $\begin{array}{l}0.93 \pm \\
0.04 \\
(1.91 \pm\end{array}$ & \\
\hline & $\begin{array}{l}(55.6 \\
\pm \\
1.31)^{\star}\end{array}$ & $\begin{array}{l}(50.3 \\
\pm \\
1.47)\end{array}$ & & & $\begin{array}{l}(3.45 \pm \\
0.19)\end{array}$ & & $0.08)$ & $0.06)$ & \\
\hline
\end{tabular}




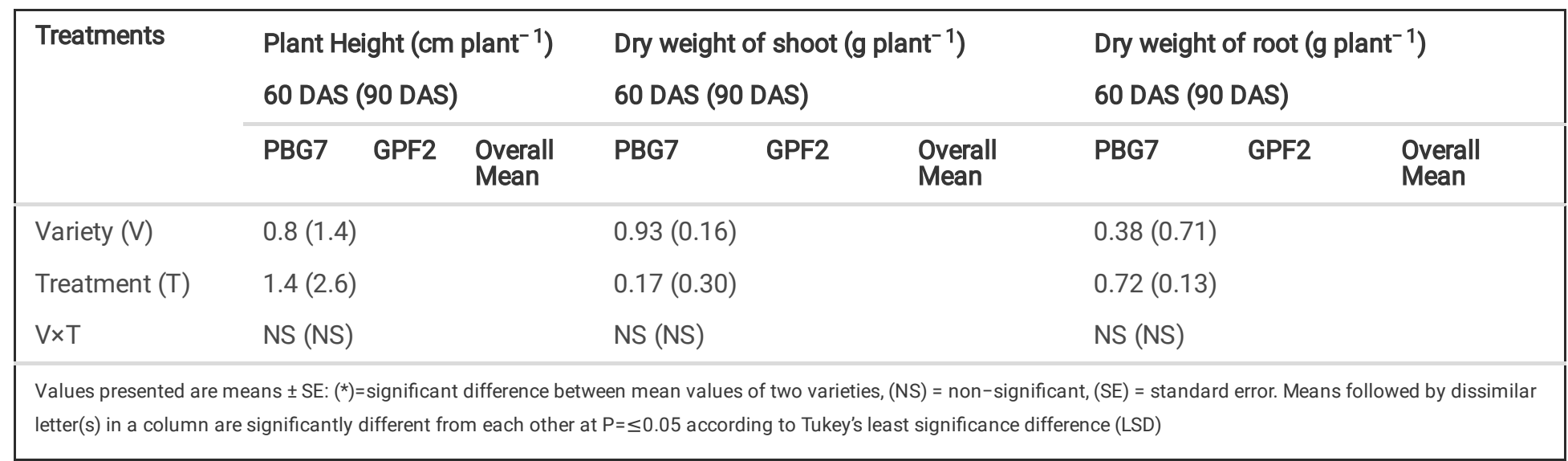

The dry weight of shoot 60 DAS, revealed significant variation among all the mono-inoculant treatments over un-inoculated control apart from the strain MR17 (Table 4), however, recommended MR33 (14.9\%) was at par with MR2 (14.0\%). Similarly, dual-inoculant treatment of RB-1 + MR2 performed at par with recommended RB-1 + MR33 for enhancement of dry weight of shoot. However, the highest increase was significantly obtained with RB-1 + MR2 (18.0\%) over MR2 alone followed by recommended RB-1 + MR33 (15.4\%) over MR33 alone treatment. The effect of treatments on varieties was not significant, while both the chickpea varieties were at par for enhancement of dry weight of shoot. Similar trend was observed at 90 DAS, where the highest dry weight of shoot was significantly recorded with strain MR2 (18.4\%) followed by recommended strain MR33 (16.5\%) over un-inoculated control. Dual-inoculant treatment of RB-1 + MR2 and recommended RB-1 + MR33 led to significantly enhanced dry weight of shoot by $34.1 \%$ and $31.6 \%$, respectively over un-inoculated control. Further, RB-1 + MR2 (13.2\%) and recommended RB-1 + MR33 (12.9\%) significantly increased the dry weight of shoot over MR2 and MR33 alone treatments, respectively. Both chickpea varieties viz PBG7 and GPF2 were at par for the dry weight of shoot at 90 DAS.

In terms of the dry weight of root at 60 DAS, significant differences were not observed among all the treatments. However, at 90 DAS, all the mono-inoculant treatments resulted in significantly improved root dry weight over un-inoculated control apart from Mesorhizobium sp. strain MR17 (Table 4). The increase was witnessed with strain MR2 (8.7\%) followed by recommended MR33 (7.6\%) over uninoculated control. All the dual-inoculant treatments significantly increased the dry weight of root ranged from $17.4 \%-24.5 \%$ over uninoculated control. However, recommended RB-1 + MR33 (14.6\%) produced the highest root dry weight over MR33 alone followed by RB1 + MR2 (14.5\%) over MR2 alone and RB-1 + MR17 (14.3\%) over MR17 alone.

For improvement of the chlorophyll content, all the treatments were at par with each other at 60 DAS (Fig. 3a). Further at 90 DAS, among all the treatments, significant enhancement in chlorophyll content was seen with RB-1 + MR2 (26.6\%) and recommended RB-1 + MR33 (23.8\%) over un-inoculated control. However, RB-1 + MR2 and recommended RB-1 + MR33 were at par with each other and also with MR2 and MR33 alone treatments, respectively. The pooled mean analysis also revealed a significant interaction between the varieties and the treatments. However, chickpea variety PBG7 significantly increased the chlorophyll content by $13.7 \%$ over GPF2 variety. For carotenoid content of leaves at 60 and 90 DAS, all treatments were at par, and the effect of treatments on varieties was non-significant (Fig. 3b). However, dual-inoculant treatments increased carotenoid content by 1.1 fold over the mono-inoculant treatments.

\subsubsection{Symbiotic nitrogen fixation}

The number of nodules (NN) at 60 and 90 DAS was significantly affected by all mono-inoculant treatments except Mesorhizobium sp. strain MR17 in the test chickpea varieties (Table 5). At 60 DAS, a significantly high number of nodules were observed in mono-inoculant treatments as compared to un-inoculated control. The highest number of nodules was registered with RB-1 + MR2 (35 NN plant $\left.{ }^{-1}\right)$ over $^{-1}$ un-inoculated control. Also, an extra abundant number of nodules were obtained with recommended RB-1 + MR33 (13.8\%) and RB-1 + MR2 (12.9\%) over MR33 and MR2 alone treatments, respectively. Further, at 90 DAS, a significantly high number of nodules were observed with recommended strain MR33 (21.9\%) and MR2 (14.6\%) alone treatments over un-inoculated control. Among dual-inoculant treatments, the highest number of nodules were significantly recorded with recommended RB-1 + MR33 (36.6\%) whereas the least was recorded with RB-1 + MR17 (14.6\%) over un-inoculated control. Moreover, a significant increase was observed with recommended RB-1 + MR33 (12.0\%), RB-1 + MR2 (12.8\%) and RB-1 + MR17 (9.3\%) over MR33, MR2 and MR17 alone treatments, respectively. Also, PBG7 Variety significantly increased the nodule number over GPF2 variety at 60 DAS (14.3\%) and 90 DAS (20.5\%). 
Table 5

Effect of potential pesticide tolerant strains of Mesorhizobium sp. and rhizobacterium RB-1 on nodulation

\begin{tabular}{|c|c|c|c|c|c|c|c|c|c|}
\hline \multirow[t]{3}{*}{ Treatments } & \multirow{2}{*}{\multicolumn{3}{|c|}{$\begin{array}{l}\text { Number of nodules (NN } \\
\text { plant }^{-1} \text { ) } \\
60 \text { DAS ( } 90 \text { DAS) }\end{array}$}} & \multirow{2}{*}{\multicolumn{3}{|c|}{$\begin{array}{l}\text { Dry weight of nodules (mg plant }{ }^{-1} \text { ) } \\
60 \text { DAS (90 DAS) }\end{array}$}} & \multirow{2}{*}{\multicolumn{3}{|c|}{$\begin{array}{l}\text { Leghaemoglobin content ( } \mathrm{mg} \mathrm{g}^{-1} \text { fresh } \\
\text { weight of nodules) } \\
60 \text { DAS ( } 90 \text { DAS) }\end{array}$}} \\
\hline & & & & & & & & & \\
\hline & PBG7 & GPF2 & $\begin{array}{l}\text { Overall } \\
\text { Mean }\end{array}$ & PBG7 & GPF2 & $\begin{array}{l}\text { Overall } \\
\text { Mean }\end{array}$ & PBG7 & GPF2 & $\begin{array}{l}\text { Overall } \\
\text { Mean }\end{array}$ \\
\hline Control & $\begin{array}{l}27 \pm 3 \\
(45 \pm \\
5)\end{array}$ & $\begin{array}{l}23 \pm \\
3(36 \\
\pm 5)\end{array}$ & $\begin{array}{l}25 \pm 2 a \\
(36 \pm \\
4 a)\end{array}$ & $\begin{array}{l}136 \pm 11.9 \\
(186 \pm \\
20.0)\end{array}$ & $\begin{array}{l}126 \pm \\
10.4 \\
(161 \pm \\
19.6)\end{array}$ & $\begin{array}{l}131 \pm 7.7 a \\
(174 \pm \\
13.9 a)\end{array}$ & $\begin{array}{l}2.03 \pm \\
0.13(3.01 \\
\pm 0.18)\end{array}$ & $\begin{array}{l}1.93 \pm \\
0.14 \\
(2.93 \pm \\
0.15)\end{array}$ & $\begin{array}{l}1.98 \pm 0.09 a \\
(2.97 \pm \\
0.11 a)\end{array}$ \\
\hline MR33 & $\begin{array}{l}31 \pm 4 \\
(55 \pm \\
6)\end{array}$ & $\begin{array}{l}26 \pm \\
4(45 \\
\pm 5)\end{array}$ & $\begin{array}{l}29 \pm 3 b \\
(45 \pm \\
4 b)\end{array}$ & $\begin{array}{l}170 \pm 14.5 \\
(225 \pm \\
17.8)\end{array}$ & $\begin{array}{l}135 \pm 8.5 \\
(184 \pm \\
20.4)\end{array}$ & $\begin{array}{l}152 \pm 9.6 b \\
(205 \pm \\
11.8 b)\end{array}$ & $\begin{array}{l}2.38 \pm \\
0.18(3.60 \\
\pm 0.19)\end{array}$ & $\begin{array}{l}2.24 \pm \\
0.15 \\
(3.22 \pm \\
0.20)\end{array}$ & $\begin{array}{l}2.31 \pm 0.11 b \\
(3.41 \pm \\
0.15 a)\end{array}$ \\
\hline MR2 & $\begin{array}{l}33 \pm 3 \\
(51 \pm \\
6)\end{array}$ & $\begin{array}{l}29 \pm \\
3(44 \\
\pm 4)\end{array}$ & $\begin{array}{l}31 \pm \\
2 \mathrm{bc} \\
(44 \pm \\
4 \mathrm{bc})\end{array}$ & $\begin{array}{l}161 \pm 16.5 \\
(221 \pm \\
15.4)\end{array}$ & $\begin{array}{l}134 \pm 5.6 \\
(171 \pm \\
14.4)\end{array}$ & $\begin{array}{l}147 \pm 9.2 \mathrm{bc} \\
(196 \pm \\
15.9 \mathrm{bc})\end{array}$ & $\begin{array}{l}2.31 \pm \\
0.17(3.51 \\
\pm 0.19)\end{array}$ & $\begin{array}{l}2.15 \pm \\
0.13 \\
(3.21 \pm \\
0.13)\end{array}$ & $\begin{array}{l}2.23 \pm \\
0.10 \mathrm{bc} \\
(3.36 \pm \\
0.12 \mathrm{a})\end{array}$ \\
\hline MR17 & $\begin{array}{l}29 \pm 3 \\
(48 \pm \\
5)\end{array}$ & $\begin{array}{l}23 \pm \\
4(37 \\
\pm 6)\end{array}$ & $\begin{array}{l}26 \pm 2 a \\
(37 \pm \\
4 a)\end{array}$ & $\begin{array}{l}152 \pm 15.8 \\
(204 \pm \\
25.3)\end{array}$ & $\begin{array}{l}116 \pm 7.6 \\
(178 \pm \\
14.9)\end{array}$ & $\begin{array}{l}134 \pm 9.9 a \\
(191 \pm \\
13.5 \mathrm{~cd})\end{array}$ & $\begin{array}{l}2.10 \pm \\
0.17(3.44 \\
\pm 0.17)\end{array}$ & $\begin{array}{l}1.99 \pm \\
0.11 \\
(3.21 \pm \\
0.16)\end{array}$ & $\begin{array}{l}2.04 \pm 0.10 a \\
(3.33 \pm \\
0.12 a)\end{array}$ \\
\hline $\begin{array}{l}\text { RB-1 + MR33 } \\
\text { (Recommended) }\end{array}$ & $\begin{array}{l}35 \pm 4 \\
(61 \pm \\
8)\end{array}$ & $\begin{array}{l}31 \pm \\
3(51 \\
\pm 6)\end{array}$ & $\begin{array}{l}33 \pm 3 d \\
(51 \pm \\
5 d)\end{array}$ & $\begin{array}{l}185 \pm 19.2 \\
(245 \pm \\
22.1)\end{array}$ & $\begin{array}{l}161 \pm \\
12.0 \\
(216 \pm \\
17.5)\end{array}$ & $\begin{array}{l}173 \pm 11.4 d \\
(230 \pm \\
14.2 \mathrm{e})\end{array}$ & $\begin{array}{l}2.66 \pm \\
0.19(3.94 \\
\pm 0.18)\end{array}$ & $\begin{array}{l}2.42 \pm \\
0.17 \\
(3.73 \pm \\
0.20)\end{array}$ & $\begin{array}{l}2.54 \pm 0.12 d \\
(3.83 \pm \\
0.13 a)\end{array}$ \\
\hline RB-1 + MR2 & $\begin{array}{l}37 \pm 5 \\
(58 \pm \\
5)\end{array}$ & $\begin{array}{l}34 \pm \\
4(48 \\
\pm 5)\end{array}$ & $\begin{array}{l}35 \pm 3 e \\
(48 \pm \\
4 b c e)\end{array}$ & $\begin{array}{l}178 \pm 17.4 \\
(237 \pm \\
23.2)\end{array}$ & $\begin{array}{l}154 \pm \\
11.3 \\
(211 \pm \\
16.6)\end{array}$ & $\begin{array}{l}166 \pm \\
10.5 \mathrm{de} \\
(224 \pm \\
14.2 \mathrm{ef})\end{array}$ & $\begin{array}{l}2.58 \pm \\
0.17(3.83 \\
\pm 0.20)\end{array}$ & $\begin{array}{l}2.29 \pm \\
0.17 \\
(3.71 \pm \\
0.21)\end{array}$ & $\begin{array}{l}2.43 \pm \\
0.13 \mathrm{de} \\
(3.77 \pm 0.14)\end{array}$ \\
\hline \multirow[t]{2}{*}{ RB-1 + MR17 } & $\begin{array}{l}34 \pm 4 \\
(51 \pm \\
6)\end{array}$ & $\begin{array}{l}31 \pm \\
4(44 \\
\pm 6)\end{array}$ & $\begin{array}{l}33 \pm 3 d \\
(44 \pm \\
4 f)\end{array}$ & $\begin{array}{l}178 \pm 12.3 \\
(223 \pm \\
27.2)\end{array}$ & $\begin{array}{l}140 \pm \\
11.8 \\
(200 \pm \\
11.2)\end{array}$ & $\begin{array}{l}159 \pm 9.9 \text { ef } \\
(211 \pm \\
14.5 \mathrm{~g})\end{array}$ & $\begin{array}{l}2.36 \pm \\
0.14(3.75 \\
\pm 0.21)\end{array}$ & $\begin{array}{l}2.25 \pm \\
0.14 \\
(3.52 \pm \\
0.16)\end{array}$ & $\begin{array}{l}2.31 \pm \\
0.09 \mathrm{ef}(3.64 \\
\pm 0.13)\end{array}$ \\
\hline & $\begin{array}{l}32 \pm \\
4^{\star} \pm \\
(53 \pm \\
\left.6^{*}\right)\end{array}$ & $\begin{array}{l}28 \pm \\
4(44 \\
\pm 5)\end{array}$ & & $\begin{array}{l}166 \pm \\
15.4^{\star} \\
(220 \pm \\
\left.21.6^{\star}\right)\end{array}$ & $\begin{array}{l}138 \pm 9.6 \\
(188 \pm \\
16.4)\end{array}$ & & $\begin{array}{l}2.34 \pm \\
0.16^{\star} \\
(3.58 \pm \\
0.19)\end{array}$ & $\begin{array}{l}2.18 \pm \\
0.14 \\
(3.36 \pm \\
0.18)\end{array}$ & \\
\hline Variety (V) & \multicolumn{3}{|c|}{$1.15(2.31)$} & \multicolumn{3}{|l|}{$5.9(5.5)$} & \multicolumn{3}{|l|}{$0.77(0.51)$} \\
\hline Treatment $(\mathrm{T})$ & \multicolumn{3}{|c|}{$2.15(4.33)$} & \multicolumn{3}{|l|}{$11(10.3)$} & \multicolumn{3}{|l|}{$0.14(0.96)$} \\
\hline$V \times T$ & \multicolumn{3}{|c|}{ NS (NS) } & \multicolumn{3}{|l|}{ NS (NS) } & \multicolumn{3}{|l|}{ NS (0.13) } \\
\hline
\end{tabular}

Nodule biomass indicates the development of nodules. At 60 DAS, the dry weight of nodules was significantly affected by the monoinoculant treatments apart from MR17 (Table 5 and Plates 6a-d). The highest dry weight of nodules was recorded with recommended RB-1 + MR33 (32.1\%) and the least with RB-1 + MR17 (21.4\%) over the un-inoculated control. Moreover, there was a significant enhancement in dry weight of nodules with recommended RB-1 + MR33 (13.8\%), RB-1 + MR2 (12.9\%) and RB-1 + MR17 (18.7\%) over MR33, MR2 and MR17 alone treatments. At 90 DAS, significant variation was found among all treatments. Recommended RB-1 + MR33 (32.2\%) showed the highest increase in the dry weight of nodules over the control while it was at par with RB-1 + MR2 (28.7\%). The dry weight of nodules further significantly enhanced with RB-1 + MR33 over MR33 (12.2\%) followed by over RB-1 + MR2 (14.3\%) over MR2 alone treatment, respectively (Plate $6 \mathrm{~d}$ and $6 \mathrm{e}$ ). Variety PBG7 performed significantly better over GPF2 variety at 60 (7.9\%) and 90 DAS (17.0\%). 
Leghaemoglobin content was also significantly affected by all the treatments except with Mesorhizobium sp. MR17 alone over uninoculated control (Table 5). However, strain MR2 (16.7\%) was at par with the recommended strain MR33 (12.6\%) for increasing the leghaemoglobin content. Recommended RB-1 + MR33 (28.3\%) recorded the highest leghaemoglobin content whereas it was at par with MR33 alone treatment. However, RB-1 + MR2 significantly increased the leghaemoglobin content by $8.9 \%$ over MR2 alone treatment. At 90 DAS, the maximum amount of leghaemoglobin was obtained with recommended strain MR33 which further increased on dual inoculation with RB-1 as compared to un-inoculated control. However, recommended RB-1 + MR33 and RB-1 + MR2 were at par with MR33 and MR2 alone treatments, respectively. Nodulation parameters implied that the response of MR2 and recommended Mesorhizobium strain MR33 in the enhancement of SNF was better over the strain MR17. However, dual-inoculant treatments provided an added benefit in overall plant's performance.

\subsubsection{Antioxidant enzyme activities}

All the treatments significantly increased the SOD and CAT activities over the un-inoculated control (Figs. 4a and 4b). The highest increase in SOD activity was recorded with Mesorhizobium sp. strain MR2 (1.3 fold) and the lowest with Mesorhizobium sp. strain MR17 (1.2 fold) over un-inoculated control. However, SOD activity further enhanced by 1.5 fold with dual-inoculant treatment RB-1 + MR2 followed by a 1.4 fold increase with recommended RB-1 + MR33 over un-inoculated control. In congruence with SOD activity, significant enhancement in CAT activity was recorded with dual-inoculant treatments viz RB-1 + MR2 and recommended RB-1 + MR33 (1.08 fold each) over Mesorhizobium alone treatments viz MR2 and MR33. PBG7 variety demonstrated significantly high SOD and CAT activities (1.2 and 1.1 fold, respectively) over GPF2. These results showed that antioxidant enzyme activities were affected more significantly by the Mesorhizobium sp. strain MR2 as compared to recommended MR33 in mono-and dual-inoculant treatments. This presented strain MR2 as a potential candidate for improvement of plant growth and productivity in chickpea under field conditions. This can be explored as an alternative to the recommended MR33 strain in the formulation of biofertilizers in chickpea.

\subsubsection{Soil health}

In terms of dehydrogenase activity (Fig. 5a), all treatments differed significantly with un-inoculated control except MR17. However, the recommended strain MR33 (16.1\%) and strain MR2 (15.7\%) performed at par for improving soil health. The highest effect of dualinoculant treatments on soil dehydrogenase activity was found with RB-1 + MR2 (33.1\%) followed by recommended RB-1 + MR33 (29.7\%) while the least was recorded with RB-1 + MR17 (14.2\%). Further, recommended RB-1 + MR33 (11.7\%) and RB-1 + MR2 (15.0\%) significantly increased soil dehydrogenase activity over MR33 and MR2 alone treatments, respectively. Variety PBG7 performed significantly better by $16.3 \%$ over variety GPF2. On the contrary, the strain MR2 performed at par with the recommended strain MR33 for enhancement in soil urease activity at 90 DAS (Fig. 5b). Also, the highest soil urease activity was witnessed with dual-inoculant treatment of strain MR2 with RB-1 $(448 \mu \mathrm{g})$ followed by recommended RB-1 + MR33 $(440 \mu \mathrm{g})$ over un-inoculated control $(361 \mu \mathrm{g})$ while the least was recorded with RB-1 + MR17 (426 $\mu$ g). Further, RB-1 + MR2 and recommended RB-1 + MR33 significantly increased the urease activity by $7.4 \%$ and $5.3 \%$ over MR2 and MR33 alone treatments, respectively. PBG7 variety recorded significantly high urease activity (2.5\%) over GPF2 variety. This also indicated that the strain MR2 affected the soil enzyme activities closely followed by recommended strain MR33 with improvement on dual inoculation with RB-1.

\subsubsection{Total protein content of seeds, yield attributing traits and grain yield}

In terms of the number of pods, significant differences existed among all the treatments except MR17 compared to the un-inoculated control (Table 6). All the dual-inoculant treatments were at par with each other, however, an extra abundant number of pods were registered with RB-1 + MR2 (17.5\%) and recommended RB-1 + MR33 (12.7\%) over viz. MR2 and MR33 alone treatments, respectively. Mono-inoculant treatments with the recommended strain MR33 was at par with the Mesorhizobium sp. strain MR2. The performance of PBG7 variety for the number of pods was significantly better (10.3\%) over GPF2 variety. 
Table 6

Effect of potential pesticide tolerant strains of Mesorhizobium sp. and rhizobacterium RB-1 on yield attributing traits and grain yield

\begin{tabular}{|c|c|c|c|c|c|c|c|c|c|c|c|c|}
\hline \multirow[t]{2}{*}{ Treatments } & \multicolumn{3}{|c|}{ Number of Pods plant ${ }^{-1}$} & \multicolumn{3}{|c|}{ Number of Seeds pod-1 } & \multicolumn{3}{|c|}{ Hundred seed weight (g) } & \multicolumn{3}{|c|}{ Grain yield $\left(\mathrm{Kg} \mathrm{ha}^{-1}\right)$} \\
\hline & PBG7 & GPF2 & $\begin{array}{l}\text { Overall } \\
\text { Mean }\end{array}$ & PBG7 & GPF2 & $\begin{array}{l}\text { Overall } \\
\text { Mean }\end{array}$ & PBG7 & GPF2 & $\begin{array}{l}\text { Overall } \\
\text { Mean }\end{array}$ & PBG7 & GPF2 & $\begin{array}{l}\text { Overall } \\
\text { Mean }\end{array}$ \\
\hline Control & $\begin{array}{l}36 \pm \\
2.2\end{array}$ & $\begin{array}{l}33 \pm \\
2.1\end{array}$ & $\begin{array}{l}35 \pm \\
1.5 a\end{array}$ & $\begin{array}{l}1.63 \\
\pm \\
0.14\end{array}$ & $\begin{array}{l}1.65 \\
\pm \\
0.11\end{array}$ & $\begin{array}{l}1.64 \pm \\
0.09 a\end{array}$ & $\begin{array}{l}13.8 \\
\pm \\
0.83\end{array}$ & $\begin{array}{l}12.8 \\
\pm \\
0.26\end{array}$ & $\begin{array}{l}13.3 \pm \\
0.45 a\end{array}$ & $\begin{array}{l}1657 \\
\pm \\
51.70\end{array}$ & $\begin{array}{l}1557 \\
\pm \\
40.25\end{array}$ & $\begin{array}{l}1607 \pm \\
36.34 a\end{array}$ \\
\hline MR33 & $\begin{array}{l}43 \pm \\
3.4\end{array}$ & $\begin{array}{l}38 \pm \\
2.4\end{array}$ & $\begin{array}{l}40 \pm \\
2.1 \mathrm{bc}\end{array}$ & $\begin{array}{l}1.99 \\
\pm \\
0.15\end{array}$ & $\begin{array}{l}1.83 \\
\pm \\
0.17\end{array}$ & $\begin{array}{l}1.91 \pm \\
0.11 \mathrm{~b}\end{array}$ & $\begin{array}{l}15.1 \\
\pm \\
0.77\end{array}$ & $\begin{array}{l}13.4 \\
\pm \\
0.24\end{array}$ & $\begin{array}{l}14.3 \pm \\
0.46 \mathrm{~b}\end{array}$ & $\begin{array}{l}1792 \\
\pm \\
42.63\end{array}$ & $\begin{array}{l}1652 \\
\pm \\
34.76\end{array}$ & $\begin{array}{l}1722 \pm \\
35.30 \mathrm{c}\end{array}$ \\
\hline MR2 & $\begin{array}{l}43 \pm \\
3.8\end{array}$ & $\begin{array}{l}38 \pm \\
1.9\end{array}$ & $\begin{array}{l}40 \pm \\
2.2 \mathrm{bc}\end{array}$ & $\begin{array}{l}1.91 \\
\pm \\
0.18\end{array}$ & $\begin{array}{l}1.77 \\
\pm \\
0.10\end{array}$ & $\begin{array}{l}1.84 \pm \\
0.10 \mathrm{bc}\end{array}$ & $\begin{array}{l}15.7 \\
\pm \\
0.79\end{array}$ & $\begin{array}{l}14.0 \\
\pm \\
0.24\end{array}$ & $\begin{array}{l}14.9 \pm \\
0.47 c\end{array}$ & $\begin{array}{l}1784 \\
\pm \\
39.37\end{array}$ & $\begin{array}{l}1639 \\
\pm \\
33.52\end{array}$ & $\begin{array}{l}1711 \pm \\
34.55 \mathrm{~cd}\end{array}$ \\
\hline MR17 & $\begin{array}{l}39 \pm \\
3.7\end{array}$ & $\begin{array}{l}36 \pm \\
2.6\end{array}$ & $\begin{array}{l}37 \pm \\
2.2 \mathrm{~b}\end{array}$ & $\begin{array}{l}1.79 \\
\pm \\
0.13\end{array}$ & $\begin{array}{l}1.72 \\
\pm \\
0.13\end{array}$ & $\begin{array}{l}1.75 \pm \\
0.09 \mathrm{bd}\end{array}$ & $\begin{array}{l}14.7 \\
\pm \\
0.68\end{array}$ & $\begin{array}{l}13.1 \\
\pm \\
0.30\end{array}$ & $\begin{array}{l}13.9 \pm \\
0.43 \mathrm{ab}\end{array}$ & $\begin{array}{l}1655 \\
\pm \\
58.01\end{array}$ & $\begin{array}{l}1621 \\
\pm \\
36.27\end{array}$ & $\begin{array}{l}1638 \pm \\
34.19 a b\end{array}$ \\
\hline $\begin{array}{l}\text { RB-1 + MR33 } \\
\text { (Recommended) }\end{array}$ & $\begin{array}{l}48 \pm \\
3.0\end{array}$ & $\begin{array}{l}42 \pm \\
1.8\end{array}$ & $\begin{array}{l}45 \pm \\
1.9 \mathrm{~d}\end{array}$ & $\begin{array}{l}2.14 \\
\pm \\
0.16\end{array}$ & $\begin{array}{l}1.91 \\
\pm \\
0.14\end{array}$ & $\begin{array}{l}2.02 \pm \\
0.11 \mathrm{e}\end{array}$ & $\begin{array}{l}15.7 \\
\pm \\
0.82\end{array}$ & $\begin{array}{l}13.4 \\
\pm \\
0.27\end{array}$ & $\begin{array}{l}14.6 \pm \\
0.54 \mathrm{bd}\end{array}$ & $\begin{array}{l}1874 \\
\pm \\
29.01\end{array}$ & $\begin{array}{l}1711 \\
\pm \\
38.87\end{array}$ & $\begin{array}{l}1792 \pm \\
33.43 f\end{array}$ \\
\hline RB-1 + MR2 & $\begin{array}{l}50 \pm \\
3.1\end{array}$ & $\begin{array}{l}44 \pm \\
2.1\end{array}$ & $\begin{array}{l}47 \pm \\
2.0 \mathrm{de}\end{array}$ & $\begin{array}{l}2.20 \\
\pm \\
0.16\end{array}$ & $\begin{array}{l}2.04 \\
\pm \\
0.15\end{array}$ & $\begin{array}{l}2.12 \pm \\
0.11 \mathrm{ef}\end{array}$ & $\begin{array}{l}16.1 \\
\pm \\
0.83\end{array}$ & $\begin{array}{l}14.5 \\
\pm \\
0.36\end{array}$ & $\begin{array}{l}15.3 \pm \\
0.50 \mathrm{ce}\end{array}$ & $\begin{array}{l}1864 \\
\pm \\
29.23\end{array}$ & $\begin{array}{l}1691 \\
\pm \\
38.00\end{array}$ & $\begin{array}{l}1778 \pm \\
36.16 f g\end{array}$ \\
\hline RB-1 + MR17 & $\begin{array}{l}44 \pm \\
4.2\end{array}$ & $\begin{array}{l}41 \pm \\
3.0\end{array}$ & $\begin{array}{l}42 \pm \\
2.5 \mathrm{df}\end{array}$ & $\begin{array}{l}2.04 \\
\pm \\
0.17\end{array}$ & $\begin{array}{l}1.90 \\
\pm \\
0.13\end{array}$ & $\begin{array}{l}1.97 \pm \\
0.10 \mathrm{eg}\end{array}$ & $\begin{array}{l}15.4 \\
\pm \\
0.73\end{array}$ & $\begin{array}{l}13.8 \\
\pm \\
0.15\end{array}$ & $\begin{array}{l}14.6 \pm \\
0.43 d\end{array}$ & $\begin{array}{l}1754 \\
\pm \\
30.91\end{array}$ & $\begin{array}{l}1669 \\
\pm \\
32.05\end{array}$ & $\begin{array}{l}1711 \pm \\
26.05 \mathrm{e}\end{array}$ \\
\hline Mean & $\begin{array}{l}43 \pm \\
3.3^{\star}\end{array}$ & $\begin{array}{l}39 \pm \\
2.3\end{array}$ & & $\begin{array}{l}1.96 \\
\pm \\
0.16\end{array}$ & $\begin{array}{l}1.83 \\
\pm \\
0.13\end{array}$ & & $\begin{array}{l}15.2 \\
\pm \\
0.78^{\star}\end{array}$ & $\begin{array}{l}13.6 \\
\pm \\
0.26\end{array}$ & $14.4 \pm$ & $\begin{array}{l}1769 \\
\pm \\
40.12^{\star}\end{array}$ & $\begin{array}{l}1648 \\
\pm \\
36.25\end{array}$ & \\
\hline Interactions: & 1.72 & & & 0.53 & & & 0.3 & & & 21.2 & & \\
\hline Variety (V) & 3.22 & & & 0.10 & & & 0.6 & & & 39.7 & & \\
\hline Treatment $(\mathrm{T})$ & NS & & & NS & & & NS & & & 56.1 & & \\
\hline$V \times T$ & & & & & & & & & & & & \\
\hline
\end{tabular}

The number of seeds per pod also showed significant differences for all the treatments, however, interaction between variety and treatment was non-significant (Table 6). In mono-inoculant treatments, the highest improvement in the number of pods was recorded with strain MR2 (16.5\%) while strain MR17 (6.7\%) displayed the least increase over un-inoculated control. Further, among dual-inoculant treatments, enhancement in the number of pods was highest with RB-1 + MR2 (29.3\%) followed by recommended RB-1 + MR33 (23.2\%) and RB-1 + MR17 (20.1\%) over un-inoculated control. The number of seeds further enhanced by $10.9 \%$ with RB-1 + MR2 over MR2 alone followed by $8.0 \%$ with recommended RB-1 + MR33 over MR33 alone. Means of varieties (PBG7 and GPF2) were at par for the number of pods.

All the treatments showed significant variation for the hundred seed weight except MR17 alone treatment (Table 6). However, strain MR2 (12.0\%) and the recommended strain MR33 (6.8\%) were at par with each other for the improvement of seed weight as compared to uninoculated control. The highest seed weight was observed with RB-1 + MR2 (15.0\%) followed by recommended RB-1 + MR33 (9.8\%) and RB-1 + MR17 (9.8\%) over un-inoculated control. However, all the dual-inoculant treatments were at par with individual Mesorhizobium alone treatments for improvement of the seed weight. There was a significant effect of PBG7 variety on the enhancement of seed weight by $11.8 \%$ over GPF2 variety. Protein content of seeds did not show any significant difference with the microbial treatments and the varieties. Among mono-inoculant treatments, the highest protein content was observed with MR33 (20.2\%) followed by MR2 (20.0\%) and MR17 (19.7\%) as compared to un-inoculated control (18.9\%). While, among dual-inoculant treatments, the maximum protein content 
was recorded with RB-1 + MR2 (21.4\%) followed by recommended RB-1 + MR33 (21.1\%) and RB-1 + MR17 (20.5\%). An additional increase in protein content of seed was noticed with dual-inoculants viz. RB-1 + MR2 and recommended RB-1 + MR33 by 1.04 fold each over MR2 and MR33 alone treatments, respectively. For grain yield, a significant difference was observed with all the mono-inoculant treatments except MR17 (Table 6) while recommended MR33 (7.2\%) was at par with MR2 (6.5\%) over un-inoculated control. However, in dual-inoculant treatments, significantly high grain yield was registered with recommended RB-1 + MR33 (11.5\%) followed by RB-1 + MR2 (10.6\%) and least was noticed with RB-1 + MR17 (6.5\%) over un-inoculated control. In corroboration with growth and symbiotic parameters, dual-inoculant treatments; recommended RB-1 + MR33 (4.1\%) and RB-1 + MR2 (3.8\%) significantly increased the grain yield over Mesorhizobium alone treatments viz MR33 and MR2, respectively. Grain yield in PBG7 variety was significantly affected (7.3\%) by the microbial inoculant treatments over GPF2 variety.

\section{Discussion}

The effect of pesticides on legume-rhizobia symbiosis depends on rhizobial species, type, and the concentration of pesticide (Kumar et al. 2010). Results from our in-vitro screening experiment also revealed appreciable differences among strains of Mesorhizobium sp. for tolerance to different pesticides and doses with significantly high tolerance shown in herbicides viz. pendimethalin and ready-mix followed by fungicide (carbendazim) and insecticide (chlorpyrifos). Higher tolerance with pre-emergence herbicide (pendimethalin) in our investigation might be due to the decomposition of complex $\mathrm{N}$-containing pendimethalin molecule which might have been used as a proteinaceous source providing both $\mathrm{C}$ and $\mathrm{N}$ to the microbes (Cycon and Pitrowska-seget 2016). A similar explanation might be attributed to tolerance with pre-plant herbicide ready-mix, which is a combination of pendimethalin + imazethapyr. As per the observations of Drouin et al. (2010), herbicide imazethapyr was not found to have any inhibitory effect on all the Rhizobium strains under study. However, in alignment with our study, a significant reduction in the number of rhizobia was recorded with fungicides (Captan and thiram) by Dunfield et al. (2000). Also in another study, a significant reduction in rhizobia was observed in combined seed treatment of chickpea with fungicide (Captan) and insecticides (Endosulfan and Chlorpyrifos) (Kunal and Sharma 2012). However, in our experiment, the highest inhibitory effect towards the growth of strains of Mesorhizobium sp. was presented by the insecticide (chlorpyrifos) as compared to other pesticides. Further, an overall decline in growth index with double doses of different pesticides might be due to their toxic effect mainly on the moderately and poorly tolerant strains of Mesorhizobium sp.

The rhizosphere is a zone of intense molecular interactions due to deposition of the root exudates, that supports higher microbial growth than the surrounding soil known as the "rhizosphere effect" (Badri et al. 2009). The influence of bacteria in the rhizosphere is largely due to the production of auxin phytohormones (indolic compounds) which depends on L-tryptophan as the main precursor (Moreira et al. 2016). Etesami et al. (2015) indicated the importance of IAA in nodulation events as it helps to circumvent the plant defence system for successful host colonization. Therefore, IAA production becomes one of the important parameters for assessing the effectiveness of rhizobia in plant growth promotion and nodulation. It has been reported by Raut et al. (2017) that IAA production varies from bacteria to bacteria and pesticide to pesticide. Further, Kulandaivel and Nagarajan (2014) confirmed that the strains of Pseudomonas and Azospirillum produced IAA for up to $1.25 \%$ concentration of pesticide endosulfan while Klebsiella strain inhibited the IAA production. Similar variations in the IAA production by Mesorhizobium strains with different pesticides in our study might be attributed to inhibition or change in IAA producing metabolic pathways by the pesticides. Moreover, a significant increase in the IAA production with tryptophan supplementation in all the treatments of Mesorhizobium strains with pesticides as well as control treatment without pesticides was attributed to the utilization of tryptophan as the precursor for IAA biosynthesis. Further, on an overall basis, among different pesticides, a significant increase in the IAA production with pendimethalin treatment as compared to the control might be attributed to the utilization of pendimethalin as a substrate for the production of energy used for the synthesis of IAA. However, a significant reduction in the IAA production with the ready-mix, followed by chlorpyrifos and carbendazim as compared to the control could be due to the imposition of stress or toxic effect of pesticides on biosynthetic machinery of Mesorhizobium strains for production of important biomolecules like IAA. Such a variation of IAA in Mesorhizobium strains with different pesticides is well supported by the study of Drouin et al. (2010), who noticed different effects of various classes of pesticides (insecticides, herbicides and fungicides) on rhizobia.

Iron $(\mathrm{Fe})$ is an essential micronutrient for plants and microorganisms, involved in various important biological processes, such as photosynthesis, respiration, chlorophyll biosynthesis and SNF. Bacteria can overcome the nutritional Fe limitation by using chelating agents called siderophores that form a complex with $\mathrm{Fe}^{3+}$ which may be absorbed by plant species for partial Fe nutrition. Competition for iron acquisition among rhizobia determines successful persistence in the rhizosphere leading to efficient host colonization (Raines et al. 2016). For siderophore production, under pesticide stress in our study, pesticide tolerant Mesorhizobium sp. strains MR2 was observed to be more promising followed by recommended strain MR33 and strain MR17. Our study is in confirmation with the work of Ahemad and Khan (2012) who reported siderophores-production by Mesorhizobium sp. strain MRC4 on CAS agar medium amended 
with insecticides (fipronil and pyriproxyfen). Cell wall degrading enzymes like the protease and cellulase are produced by rhizobia which play a role in the degradation of the plant cell wall for infection thread formation and also in antagonism against pathogenic fungi. In congruence with our findings, protease production has been reported in rhizobia strains by Oliveira et al. (2010). Also, Robledo et al. (2012) purified a cell-bound bacterial cellulase $\left(\mathrm{CelC}_{2}\right)$ enzyme from Rhizobium leguminosarum bv. trifolii nodulating the clover. In contrast to these studies, we observed positive siderophore production also in presence of herbicides (pendimethalin and ready-mix). Further, we analyzed the effect of mono-inoculation of pesticide tolerant strains of Mesorhizobium sp. and their dual inoculation with the recommended culture of PGP strain rhizobacterium RB-1 along with recommended application of pesticides with in-vitro seed germination assay. Several studies have demonstrated a significant enhancement in seed germination and seedling vigour in chickpea on co-inoculation with Rhizobium and PGP bacteria (Abdel Fattah 2015; Verma et al. 2013; Kaur et al. 2015). As per our findings also, significant improvement in germination and vigor index was noticed with potential pesticide tolerant strains of Mesorhizobium sp. alone as compared to the un-inoculated control that significantly improved with individual dual inoculation treatments.

Plant height indicates the growth index of the crop. Production of PGP substances like IAA affects many physiological activities of plants including cell enlargement, cell division, root initiation, growth rate, phototropism, geotropisms, and apical dominance and also the symbiotic rhizobium-legume interaction. Liu et al. (2017) reported that effective IAA producing isolates can stimulate plant growth due to their early colonizing ability to circumvent the plant defence mechanisms. This is well in line with the present study, where monoinoculant treatments with strains of Mesorhizobium sp. resulted in increased plant height, dry weight of shoot and root over uninoculated control with a synergistic effect in dual-inoculant treatments. Pesticides produce negative effects on crop physiology - especially on photosynthesis - leading to a potential decrease in both the growth and the yield of crops as demonstrated by Giménez-Moolhuyzen et al. (2020). However, in our study significant enhancement in plant growth was noticed with all the treatments despite the application of pesticides as compared to the un-inoculated control (with recommended pesticides application alone). This was correlated to the production of IAA by the strains of Mesorhizobium sp. under pesticide stress in our in-vitro experiment. Our results are in corroboration with Ahemad and Khan (2012) who attributed the improvement in plant growth parameters and productivity in chickpea to IAA production in pesticide tolerant Mesorhizobium sp. strain MRC4. It has been confirmed by Kannan et al. (2015) that there is a positive interaction between root colonization, nutrient uptake and growth promotion of plants. In line with these finding, our results for the increased chlorophyll content with dual-inoculant treatments of Mesorhizobium strains with RB-1 are related to efficient uptake of nutrients such as $\mathrm{Mg}$ (an important part of chlorophyll molecule) and $\mathrm{N}$ and Fe (play role in photosynthesis and enzymes involved in chlorophyll synthesis). Nodulation is an important trait for effective SNF in leguminous plants, however, pesticides have been reported to trigger root hair deformations and inhibit symbiosis (Fox et al. 2007). In the present investigation, efficient nodulation caused by pesticide tolerant strains of Mesorhizobium sp. might be caused by counteraction of phytotoxic effects of pesticides by their biodegradation or enzymatic hydrolysis (Martani et al. 2011). Our results are in agreement with Ahemad and Khan (2011) for improvement of nodulation parameters (nodule number and nodule dry weight) with herbicide-tolerant Bradyrhizobium strain MRM6 with PGP traits as compared to un-inoculated plants in greengram. Moreover, in a previous study, Gonzalez et al. (1996) showed that herbicide imazethpyr did not have any direct influence on Rhizobium spp. and decline in pea nodulation was attributed to the effect on plant growth rather than on rhizobia. Similarly in our study, the intrinsic tolerance of strains of Mesorhizobium sp. towards pesticides ameliorated the harmful effects of pesticides and substantial production of PGP substances by rhizobacterium RB-1 further improved the plant growth and development by creating more infection sites. This accounted for the significant increase in nodulation with dual inoculation treatments. In line with this, Etesami et al. (2015) established that IAA production by bacteria in root nodules activates $\mathrm{H}^{+}$ATPase required for energy production in nodules. Further, Ghosh et al. (2015) correlated the production of IAA and other PGP substances by Rhizobium undicola to the plant-microbe interaction and nodule function in Neptunia oleracea. These findings are in close corroboration to our results on improved nodule biomass and $\mathrm{N}$ fixation with multifunctional pesticide tolerant strains of Mesorhizobium sp. as compared to the un-inoculated control. Synthesis of $\mathrm{O}_{2}$ scavenging leghaemoglobin is an index of efficient SNF in legumes as it maintains high levels of ATP (Diez-Mendez et al. 2015). As per our results, higher nodulation and $\mathrm{N}$ fixation with dualinoculant treatments might have increased the nodule occupancy leading to increased leghaemoglobin content over mono-inoculant treatments. Moreover, multifunctional traits of potential pesticide tolerant Mesorhizobium strains and PGP strain RB-1 viz. siderophores mediated uptake of iron (a constituent of leghaemoglobin protein) might have contributed to the increased leghaemoglobin content in dual-inoculant treatments (Gonzalez et al. 2016). Our results are well supported by Korir et al. (2017) where co-inoculation of Rhizobium and Paenibacillus polymyxa enhanced leghaemoglobin concentration, nitrogenase activity, and $\mathrm{N}$ fixation efficiency in common bean.

Application of agrochemicals like pesticides induce a significant amount of abiotic stress resulting in the production of reactive oxygen species (ROS) that leads to the disruption of cellular activities. Superoxide dismutase enzyme (SOD) converts superoxide anion free radical $\left(\mathrm{O}_{2}\right)$ to $\mathrm{H}_{2} \mathrm{O}_{2}$. On the contrary, catalase enzyme (CAT) plays a role in converting $\mathrm{H}_{2} \mathrm{O}_{2}$ to oxygen and water. In our study, mono- 
inoculant treatments in chickpea with pesticide tolerant strains of Mesorhizobium sp. presented significantly high SOD activity against ROS formed in response to the environmental stresses generated under field conditions. This further confirmed their robustness for environmentally stressed conditions correlating with their intrinsic tolerance towards pesticides. Further, a significant increase in SOD activity with dual-inoculant treatments in our study is in congruence with Fatnassi et al. (2015) who reported enhanced SOD activity with combined inoculation of Rhizobium + PGPR as compared to the control in Vicia faba. Similarly, an increase in SOD and CAT activities has been reported in the co-inoculation of Piriformospora indica, Pseudomonas sp. and Mesorhizobium cicer over single inoculation with P.indica and Mesorhizobium alone in chickpea (Mansotra et al. 2015). In addition to this, Dong et al. (2014) observed that in petroleum contaminated soils, the co-inoculation of PGPR Serratia marcescens + AMF Glomus intraradices significantly improved the SOD activity over un-inoculated control. However, an overall decrease in catalase as compared to SOD activity in our study was attributed to more consumption of the catalase enzyme to detoxify ROS (Fatnassi et al. 2015). Soil enzymes viz urease and dehydrogenase are important indicators of soil microbial activity and play a role in nutrient $(\mathrm{N}$ and $\mathrm{H})$ recycling. Our findings for the significant increase in soil enzyme activities with pesticide tolerant strains of Mesorhizobium sp. is well supported by the work of Siczek and Lipiec (2016) where high soil urease and dehydrogenase activity in faba bean with Rhizobium over control was attributed to changes in microbial community structure and enzymatic processes in response to rhizobia. Corresponding to other growth and SNF parameters in our study, a significant increase in soil enzyme activities with all dual-inoculant treatments might be attributed to a higher root system which led to the accumulation of root exudates in the rhizosphere attracting higher microbial populations for mediating the enzyme activities. Batista et al. (2016) postulated that facilitated nutrient uptake by PGP bacteria along with phosphate solubilization (that provides $\mathrm{P}$ for ATP) and siderophores mediated uptake of iron (that acts as e carrier in nitrogen fixation reaction carried out by nitrogenase enzyme), enhanced the fixed $\mathrm{N}$ that was incorporated as proteins. Similarly, in our study, an increase in protein content with dual-inoculant treatments might be attributed to the increased availability of fixed $\mathrm{N}$ that was further explained by facilitated uptake of micronutrients such as Mo acting as a cofactor in nitrogenase enzyme. This is further correlated to IAA production, which helps in nutrient uptake with the proliferation of roots (Haris and Ahmad 2017). Similar reports on co-inoculation with Rhizobium and PGP bacteria have shown improvement in seed protein and N content in soybean (Hungria et al. 2015; Masciarelli 2014) and chickpea (Verma et al. 2013). Results on plant growth and SNF, are well in line with our study on the significant enhancement in the number of pods with dual-inoculant treatments that was attributed to an increase in the number of branches with PGP rhizobacterium RB-1. In addition to this, improved photosynthetic rates due to improvement in nutrient and water absorption in dual inoculation treatments resulted in higher nutrient assimilation in grain leading to increased seed weight and grain yield in our experiment. Similarly, a positive correlation between IAA production and grain yield was suggested by Verma et al. (2013) in chickpea. Our results are further supported by a pot experiment done by Ahmad et al (2016) where single inoculations of greengram significantly increased grain yield with Bradyrhizobium sp. and with Azotobacter sp. alone over control which further improved on dual inoculation (Bradyrhizobium + Azotobacter). Improvement in growth parameters, yield attributing traits and grain yield in different chickpea genotypes might be the result of differential interaction of variety with microbial inoculants which could be further attributed to variation in rhizospheric community structure in response to root exudates (Nasr Esfahania et al. 2016).

\section{Conclusions}

Current agricultural practices in Punjab have witnessed indiscriminate use of pesticides (including herbicides, insecticides and fungicides) due to which ecological performance of bio-inoculants may be compromised resulting in a reduction in the yield and productivity of the legumes. In the present study, in-vitro developed bacterial strains for pesticide tolerance revealed three robust strains of Mesorhizobium sp. viz. MR2 and MR17 and recommended MR33 that showed the highest plant growth promotional activities. Although, under laboratory conditions, strains MR2 and MR17 performed significantly better over the recommended strain MR33 for PGP traits, yet under field investigation, Mesorhizobium sp. strain MR2 was at par with recommended strain MR33 for enhancement of plant growth, SNF and yield in chickpea over un-inoculated control. Further dual-inoculant treatments with recommended PGP strain RB-1 significantly enhanced all the parameters. The potential pesticide tolerant strains MR2 and MR33 can be further explored as compatible dual-inoculants with recommended RB-1 for chickpea under environmentally stressed conditions (pesticide application) at multiple locations. Therefore, screening for robust multifunctional pesticide tolerant Mesorhizobium might open new avenues for improving chickpea productivity in pesticide stressed soils of Punjab. Overall, these findings postulate that bio-augmentation of chickpea with multifunctional Mesorhizobium with improved pesticide tolerance might be helpful in the formulation of effective bio-inoculants consortia in establishing successful chickpea-Mesorhizobium symbiosis. However, concerted research and development are needed to integrate engineered microorganisms in the agro-ecosystem for sustainable agriculture.

\section{Declarations}




\section{Acknowledgments}

The present research was supported by financial grant from Indian Council of Medical Research, New Delhi (Sanction No. 3/3/3/JRF/2014/HRD-7) as a part of Ph.D research work conducted on the project "Assessment of pesticide tolerance in Mesorhizobium spp. for growth, symbiosis and yield in chickpea (Cicer arietinum L.)" funded by the Ministry of Human Resource and Development, Government of India. The authors also acknowledge the technical support of Pulses Research Group, Department of Plant Breeding and Genetics, Punjab Agricultural University, Ludhiana, India for conducting this research work.

\section{Declaration of interests}

The authors declare that they have no known competing financial interestsor personal relationships that could have appeared to influence the work reported in this paper.

\section{References}

Abdel Fattah ME (2015) Genetic and biochemical characterization of some Egyptian rhizobia isolates nodulating Faba Bean. J Microb Biochem Technol 07:83-87. doi:10.4172/1948-5948.1000186

Aebi HE (1983) Catalase. In: Bergmeyer HO (ed) Methods of enzymatic analysis. Academic Press, New York (NY), pp. 273-286

Ahemad M, Khan MS (2009) Effect of insecticide-tolerant and plant growth-promoting Mesorhizobium on the performance of chickpea grown in insecticide stressed alluvial soils. J Crop Sci Biotechnol 12:217-226. https://doi.org/10.1007/s12892-009-0130-8

Ahemad M, Khan MS (2011) Effect of pesticides on plant growth promoting traits of greengram-symbiont, Bradyrhizobium sp. strain MRM6. Bull Environ Contam Toxicol 86:384-388. https://doi.org/10.1007/s00128-011-0231-1.

Ahmad F, Ahmad I, Altaf MM, Khan MS, Shouche YS (2016) Characterization of Paenibacillus durus (pnf16) a new isolate and its synergistic interaction with other isolated rhizobacteria in promoting growth and yield of chickpea. J Microbiol Biotechnol Food Sci 05:345-350. https://doi.org/10.15414/jmbfs.2016.5.4.345-350.

Anonymous (2013) Package of Practices for Rabi Crops. Punjab Agricultural University, Ludhiana.

Ansari PG, Rao DLN (2014) Differentiating indigenous soybean Bradyrhizobium and Rhizobium spp. of Indian soils. Indian J Microbiol 54:190-195. https://doi.org/10.1007/s12088-013-0430-z.

Badri DV, Weir TL, van der Lelie D, Vivanco JM (2009) Rhizosphere chemical dialogues: plant-microbe interactions.Curr Opin Biotechnol 20:642-650. https://doi.org/10.1016/j.copbio.2009.09.014

Bakker AW, Schippers B (1987) Microbial cyanide production in the rhizosphere in relation to potato yield reduction and Pseudomonas spp-mediated plant growth-stimulation. Soil Biol Biochem 19:451-457. https://doi.org/10.1016/0038-0717(87)90037-x

Batista BD, Taniguti LM, Almeida JR, Azevedo JL, Quecine MC (2016) Draft genome sequence of multitrait plant growth-promoting Bacillus sp. strain RZ2MS9. Genome Announc 4:9-10. https://doi.org/10.1128/genomeA.01402-16.

Bric JM, Bostock RM, Silverstone SE (1991) Rapid in situ assay for indoleacetic acid production by bacteria immobilized on a nitrocellulose membrane. Appl Environ Microbiol 57:535-538.

Brígido C, Glick BR, Oliveira S (2017) Survey of plant growth-promoting mechanisms in native portuguese chickpea Mesorhizobium isolates. Microb Ecol 73:900-915. https://doi.org/10.1007/s00248-016-0891-9.

Cycoń M, Piotrowska-Seget Z (2016) Pyrethroid-degrading microorganisms and their potential for the bioremediation of contaminated soils: a review. Front Microbiol. https://www.frontiersin.org/article/10.3389/fmicb.2016.01463.

Dong R, Gu L, Guo C, Xun F, Liu J (2014) Effect of PGPR Serratia marcescens BC-3 and AMF Glomus intraradices on phytoremediation of petroleum contaminated soil. Ecotoxicology (London, England) 23:674-680. https://doi.org/10.1007/s10646-014-1200-3.

Dere S, Gune T, Sivaci R (1998) Spectrophotometric determination of chlorophyll - a, b and total carotenoid contents of some algae species using different solvents. Turk J Bot 22:13-17. 
Diez-Mendez A, Menéndez E, García-Fraile P, Celador-Lera L, Rivas R, Mateos PF (2015) Rhizobium cellulosilyticum as a co-inoculant enhances Phaseolus vulgaris grain yield under greenhouse conditions. Symbiosis 67:135-141. doi:10.1007/s13199-015-0372-9

Drouin P, Sellami M, Prévost D, Fortin J, Antoun H (2010) Tolerance to agricultural pesticides of strains belonging to four genera of Rhizobiaceae. J Environ Sci Heal Part B-Pesticides Food Contam Agric Wastes 45:757-

65. https://doi.org/10.1080/03601234.2010.515168

Dunfield KE, Siciliano SD, Germida JJ (2000) The fungicides thiram and captan affect the phenotypic characteristics of Rhizobium leguminosarum strain $\mathrm{C} 1$ as determined by FAME and Biolog analyses. Biol Fertil Soils 31:303-

309. https://doi.org/10.1007/s003740050660

Nasr Esfahani M, Kusano M, Nguyen KH, Watanabe Y, Ha C Van, Saito K, Sulieman S, Herrera-Estrella L, Tran LS (2016) Adaptation of the symbiotic Mesorhizobium-chickpea relationship to phosphate deficiency relies on reprogramming of whole-plant metabolism. Proc Natl Acad Sci USA 113:610-619. https://doi.org/10.1073/pnas.1609440113

Etesami H, Alikhani HA, Hosseini HM (2015) Indole-3-acetic acid (IAA) production trait, a useful screening to select endophytic and rhizosphere competent bacteria for rice growth promoting agents. MethodsX 2:72-78. https://doi.org/10.1016/j.mex.2015.02.008

Fatnassi IC, Chiboub M, Saadani O, Jebara M, Jebara SH (2015) Impact of dual inoculation with Rhizobium and PGPR on growth and antioxidant status of Vicia faba L. under copper stress. C R Biol 338:241-254. https://doi.org/10.1016/j.crvi.2015.02.001

Finer KR, Fox L, Finer JJ (2016) Isolation and characterization of Agrobacterium strains from soil: A laboratory capstone experience. J Microbiol Biol Educ 17:444-450. https://doi.org/10.1128/jmbe.v17i3.1124.

Fox JE, Gulledge J, Engelhaupt E, Burow ME, McLachlan J A (2007) Pesticides reduce symbiotic efficiency of nitrogen-fixing rhizobia and host plants. Proc Natl Acad Sci USA. 104:10282-10287. https://doi.org/10.1073/pnas.0611710104.

Gaur YD, Sen AN (1981) Cultural and biochemical characteristics of root nodule bacteria of chickpea (Cicer arietinum L.). Zentralblatt für Bakteriologie, Parasitenkunde, Infektionskrankheiten und Hygiene. Zweite Naturwissenschaftliche Abteilung: Mikrobiologie der Landwirtschaft, der Technologie und des Umweltschutzes 136:307-316. doi:https://doi.org/10.1016/S0323-6056(81)80082-1

Giménez-Moolhuyzen M, Blom JV, Lorenzo-Mínguez P, Cabello T, Crisol-Martínez E (2020) Photosynthesis inhibiting effects of pesticides on sweet pepper leaves. Insects 11(2):69. https://doi.org/10.3390/insects11020069

Ghosh PK, Kumar De T, Maiti TK (2015) Production and metabolism of indole acetic acid in root nodules and symbiont (Rhizobium undicola) isolated from root nodule of aquatic medicinal legume Neptunia oleracea Lour. J Bot

2015:575067. https://doi.org/10.1155/2015/575067.

Gonzalez A, Gonzalez-Murua C, Royuela M (1996) Influence of imazethapyr on Rhizobium growth and its symbiosis with Pea (Pisum sativum). Weed Science 44(1):31-37.

González-Guerrero M, Escudero V, Saez A, Tejada-Jimenez M (2016) Transition metal transport in plants and associated endosymbionts: Arbuscular Mycorrhizal Fungi and Rhizobia. Front Plant Sci 7:1-21. https://doi.org/10.3389/fpls.2016.01088.

Gopalakrishnan S, Sathya A, Vijayabharathi R, Varshney RK, Gowda CLL, Krishnamurthy L (2015) Plant growth promoting rhizobia: challenges and opportunities. 3 Biotech 5:355-377. https://doi.org/10.1007/s13205-014-0241-x.

Gordon S, Weber R P (1951) The colorimetric estimation of IAA. Plant Physiol 26:192-99.

Haris Z, Ahmad I (2017) Impact of metal oxide nanoparticles on beneficial soil microorganisms and their secondary metabolites. Int J Life Sci Sci Res 3:1020-1030.

https://doi.org/10.21276/ijlssr.2017.3.3.10.

Hungria M, Nogueira MA, Araujo RS (2015) Soybean seed co-inoculation with Bradyrhizobium spp . and Azospirillum brasilense: A new biotechnological tool to improve yield and sustainability. Am J Plant Sci 6:811-817. https://doi.org/10.4236/ajps.2015.66087. 
Kannan VR, Sithara S, Chandru S (2015) Proportional analysis of leghaemoglobin concentration in various nodulating plants and intuitive Rhizobium species. Eur J Exp Biol 5:15-23.

Kaur N, Sharma P, Sharma S (2015) Co-inoculation of Mesorhizobium sp. and plant growth promoting rhizobacteria Pseudomonas sp. as bioenhancer and biofertilizer in chickpea (Cicer arietinum L.). Legume Research 38(3):367-74.

S Klosee MA, Tabatabai (2000) Urease activity of microbial biomass in soils as affected by cropping systems. Biol Fertil Soils 31:191199. https://doi.org/10.1007/s003740050645.

Korir H, Mungai NW, Thuita M, Hamba Y, Masso C (2017) Co-inoculation effect of Rhizobia and plant growth promoting rhizobacteria on common bean growth in a low phosphorus soil. Front Plant Sci 08:1-10. https://www.frontiersin.org/article/10.3389/fpls.2017.00141.

Kulandaivel S, Nagarajan S (2014) Pesticides induced alternations in plant growth hormone (iaa) in rhizobacteria. International Journal for Research in Applied Science and Engineering Technology 2:302-04.

Kumar M, Yadav V, Tuteja N, Johri A (2009) Antioxidant enzyme activities in maize plants colonized with Piriformospora indica. Microbiology 155:780-90. https://doi.org/10.1099/mic.0.019869-0.

Kumar N, Anubhuti Bora J I, Amb M K (2010) Chronic toxicity of the triazole fungicide tebuconazole on a heterocystous, nitrogenfixing rice paddy field cyanobacterium, Westiellopsis prolific Janet. J Microbiol Biotechnol Res 20:1134-39.

Kunal, Sharma P (2012) Compatibility of Mesorhizobium sp. Cicer with seed treatment of fungicide and insecticide in chickpea. Arch Agron Soil Sci 58:115-123. https://doi.org/10.1080/03650340.2010.506483.

Liu H, Carvalhais LC, Crawford M, Singh E, Dennis PG, Pieterse CMJ, Schenk PM (2017) Inner plant values: Diversity, colonization and benefits from endophytic bacteria. Frontiers in Microbiology . https://doi.org/10.3389/fmicb.2017.02552 .

Mansotra P, Sharma P, Sharma S (2015) Bioaugmentation of Mesorhizobium cicer, Pseudomonas spp. and Piriformospora indica for sustainable chickpea production Physiol Mol Biol Plants 21. https://doi.org/10.1007/s12298-015-0296-0.

Marklund S, Marklund G (1974) Involvement of the superoxide anion radical in the autoxidation of pyrogallol and a convenient assay for superoxide dismutase. Eur J Biochem 47:469-474. https://doi.org/10.1111/j.1432-1033.1974.tb03714.x.

Martani E, Margino S, Indradewa D, Supriyo A (2011) Isolation and selection of Rhizobium tolerant to pesticides and aluminum from acid soils in Indonesia. Journal TANAH TROPIKA (Journal of Tropical Soils) 16(1):47-54.

https://doi.org/10.5400/jts.2011.16.1.47 https://doi.org/10.5400/jts.2011.16.1.47.

Masciarelli O, Llanes A, Luna V (2014) A new PGPR co-inoculated with Bradyrhizobium japonicum enhances soybean nodulation.

Microbiol Res 169:609-615. https://doi.org/https://doi.org/10.1016/j.micres.2013.10.001.

McKenzie HA, Wallace HS (1954) The Kjeldahl determination of nitrogen: A critical study of digestion conditions-Temperature, Catalyst, and Oxidizing agent. Aust J Chem 7:55-70. https://doi.org/10.1071/CH9540055.

Meena RS, Kumar S, Datta R, Lal R, Vijayakumar V, Brtnicky M, Sharma MP, Yadav GS, Jhariya MK, Jangir CK, Pathan SI, Dokulilova T, Pecina V, Marfo TD (2020) Impact of agrochemicals on soil microbiota and management: A review. Land 9(2). https://doi.org/10.3390/land9020034

Moreira F S, Costa P B da, Souza R, deBeneduzi A, Lisboa BB, Vargas LK, Passaglia LMP (2016) Functional abilities of cultivable plant growth promoting bacteria associated with wheat (Triticum aestivum L.) crops. Genet Mol Biol 39:111121. https://doi.org/10.1590/1678-4685-GMB-2015-0140.

Oliveira, Arlem Nascimento De, Luiz Antonio De Oliveira, Jerusa Souza Andrade (2010) Production and some properties of crude alkaline proteases of indigenous central amazonian rhizobia strains. Brazilian Archives of Biology and Technology 53:1185-

95. https://doi.org/10.1590/S1516-89132010000500024.

Ouma EW, Asango AM, Maingi J, Njeru EM (2016) Elucidating the potential of native rhizobial isolates to improve biological nitrogen fixation and growth of common bean and soybean in smallholder farming systems of Kenya. Int $\mathrm{J}$

Page $19 / 24$ 
Agron. https://doi.org/10.1155/2016/4569241.

Porter SS, Faber-Hammond J, Montoya AP, Friesen ML, Sackos C (2019) Dynamic genomic architecture of mutualistic cooperation in a wild population of Mesorhizobium. ISME J 13:301-315. https://doi.org/10.1038/s41396-018-0266-y.

Raines DJ, Moroz OV, Blagova EV, Turkenburg JP, Wilson KS, Duhme-Klair A.-K (2016) Bacteria in an intense competition for iron: Key component of the Campylobacter jejuni iron uptake system scavenges enterobactin hydrolysis product. Proc Natl Acad Sci USA 113:5850-5855. https://doi.org/10.1073/pnas.1520829113.

Raut V, Shaikh I, Naphade B, Prashar K, Adhapure N (2017) Plant growth promotion using microbial IAA producers in conjunction with azolla: a novel approach. Chem Biol Technol Agric https://doi.org/10.1186/s40538-016-0083-3.

Robledo M, Rivera L, Jiménez-zurdo JI, Rivas R, Dazzo F, Velazquez E, Hirsch AM, Mateos PF (2012) Role of Rhizobium endoglucanase $\mathrm{CelC}_{2}$ in cellulose biosynthesis and biofilm formation on plant roots and abiotic surfaces 1-12. https://doi.org/10.1186/1475-2859-11125.

Schwyn B, Neilands JB (1987) Universal chemical assay for the detection and determination of siderophores. Anal Biochem 160:4756. https://doi.org/https://doi.org/10.1016/0003-2697(87)90612-9.

Siczek A, Lipiec J (2016) Impact of Faba bean-seed rhizobial inoculation on microbial activity in the rhizosphere soil during growing season. Int J Mol Sci 17:784. https://doi.org/10.3390/ijms17050784.

Singh N P (2018) Project Coordinater's reports In: All India Coordinated Research Project in Chickpea, Indian Institute of Pulses Research, Kanpur.

Tabatabai MA (1982) Sulfur1. In: Methods of Soil Analysis. Part 2. Chemical and Microbiological Properties, Agronomy Monograph SV 9.2. American Society of Agronomy, Soil Science Society of America, Madison, WI, pp. 501-

538. https://doi.org/10.2134/agronmonogr9.2.2ed.c28.

Verma JP, Yadav J, Tiwari KN, Kumar A (2013) Effect of indigenous Mesorhizobium spp. and plant growth promoting rhizobacteria on yields and nutrients uptake of chickpea (Cicer arietinum L.) under sustainable agriculture. Ecol Eng 51:282-

286. https://doi.org/http://dx.doi.org/10.1016/j.ecoleng.2012.12.022.

Vijayabharathi R, Gopalakrishnan S, Sathya A, Srinivas V, Sharma M (2018) Deciphering the tri-dimensional effect of endophytic Streptomyces sp. on chickpea for plant growth promotion, helper effect with Mesorhizobium ciceri and host-plant resistance induction against Botrytis cinerea. Microb Pathog 122:98-107.

https://doi.org/https://doi.org/10.1016/j.micpath.2018.06.019.

Vincent JM (1970) A manual for the pratical study of root-nodule bacteria. Oxford, Blackwells Scientific Publications.

Wilson DO, Reisenauer HM (1963) Determination of leghemoglobin in legume nodules. Anal Biochem 6:27-30.

doi:http://dx.doi.org/10.1016/0003-2697(63)90004-6

Witham P H, Dyes D F, Delvin R M (1971) Chlorophyll absorption of spectrum and quantitative

\section{Figures}




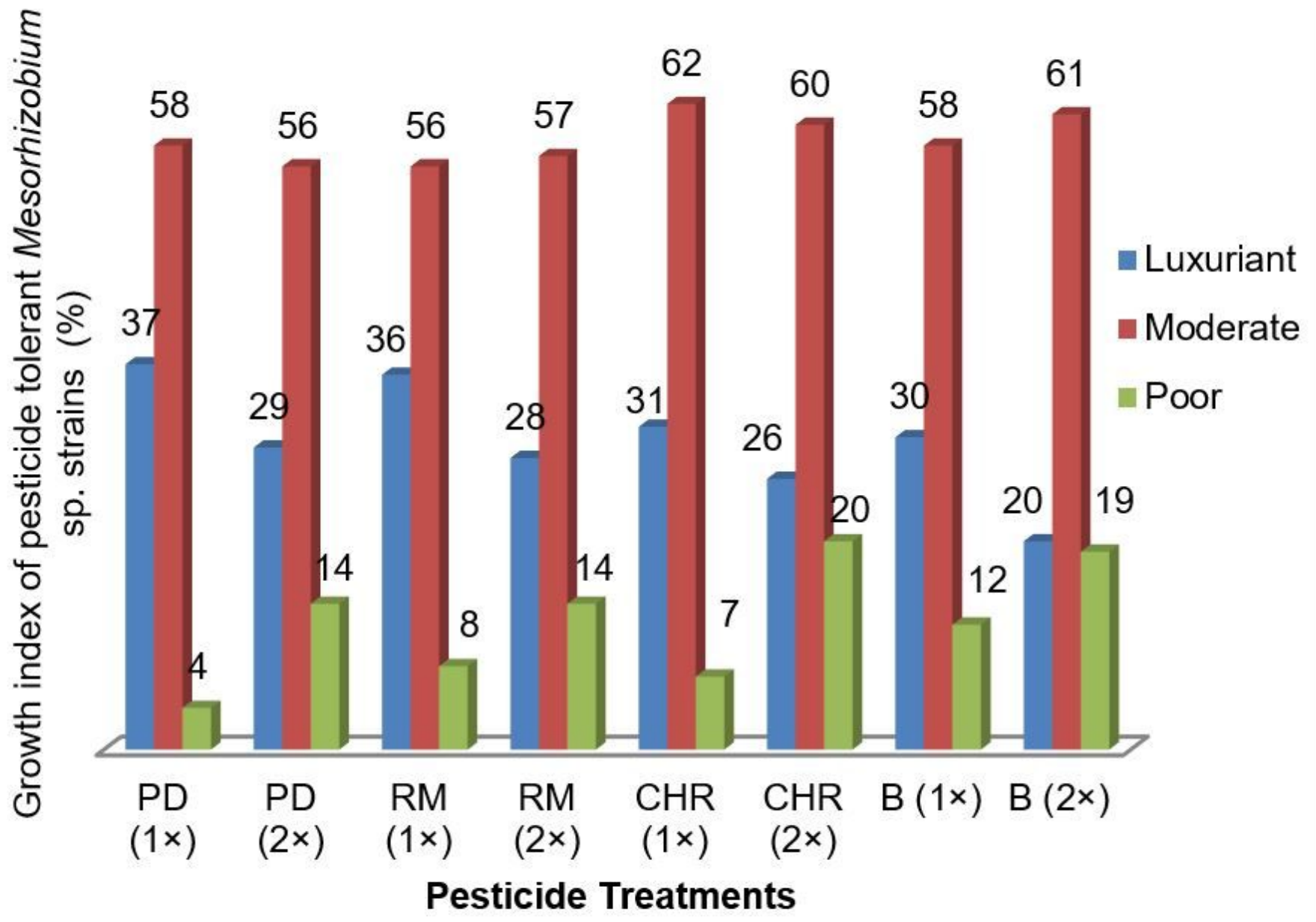

Figure 1

Growth index of strains of Mesorhizobium sp. with recommended pesticides:-pendimethalin (PD), ready mix

(pendimethalin+imazethpyr) (RM); carbendazim (B); chlorpyrifos (CHR): *Dosage of pesticides:- single dose (1x), double dose (2x) 

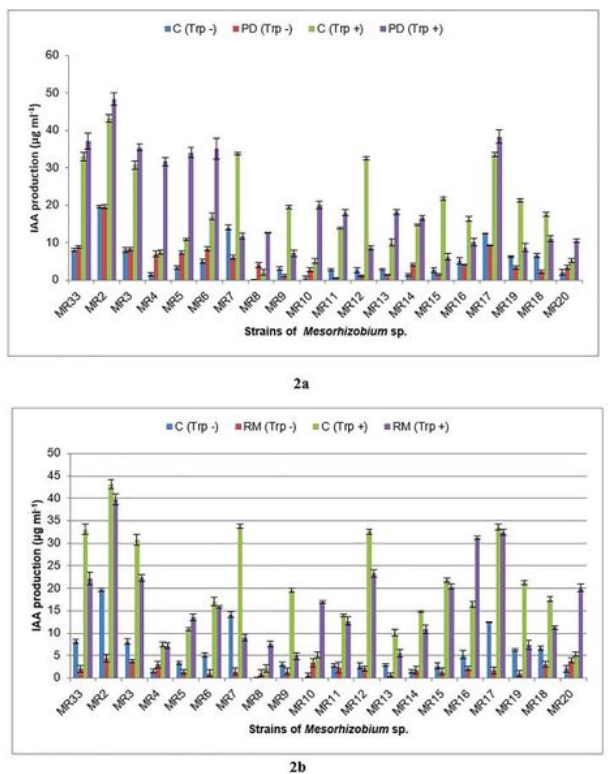

2b

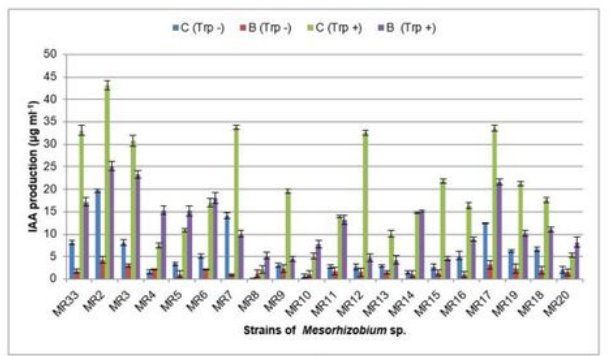

$2 \mathrm{c}$

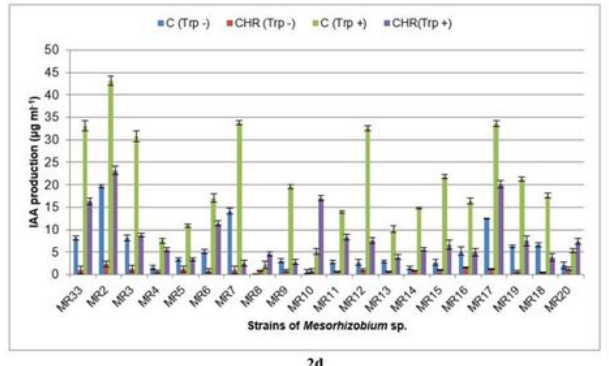

\section{Figure 2}

Indole acetic acid (IAA) production by pesticide tolerant strains of Mesorhizobium sp. with recommended doses of pesticides:- a pendimethalin (PD) b ready mix (imazethpyr+pendimethalin) (RM) c carbendazim (B) d chlorpyrifos (CHR) as compared to control treatments (C) in tryptophan's presence (Trp+) and absence (Trp-). ${ }^{*}$ The values presented are Means \pm SE. All the treatments means differed significantly with each other and also with pesticide and tryptophan treatments according to Tukey's least significance difference (LSD) at $P \leq 0.05$. 


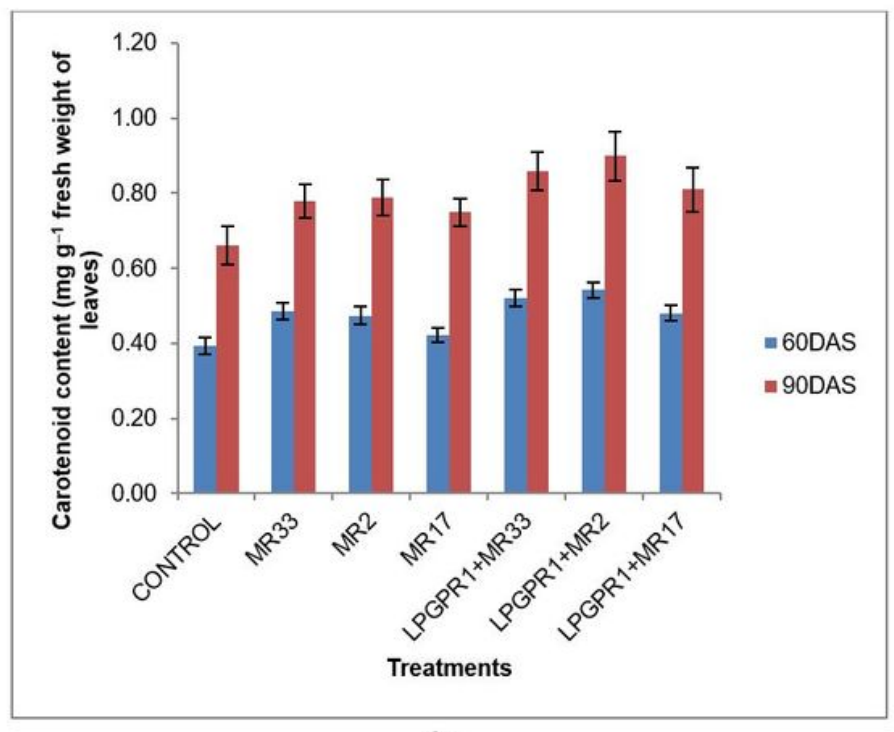

3a

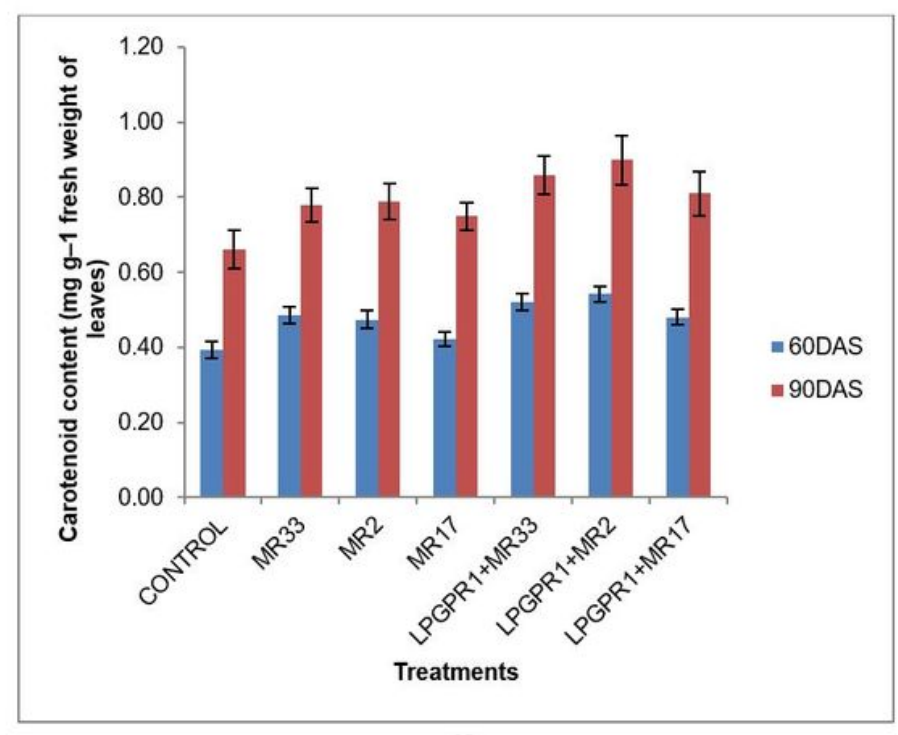

$3 \mathbf{b}$

\section{Figure 3}

Effect of potential pesticide tolerant strains of Mesorhizobium sp. and rhizobacterium RB-1 on chlorophyll and carotenoid content *Values presented are overall means of PBG and GPF2 chickpea varieties \pm SE (standard error). All the treatments means were at par with each other both at 60 and 90 DAS according to Tukey's least significance difference (LSD) at P $\leq 0.05$.

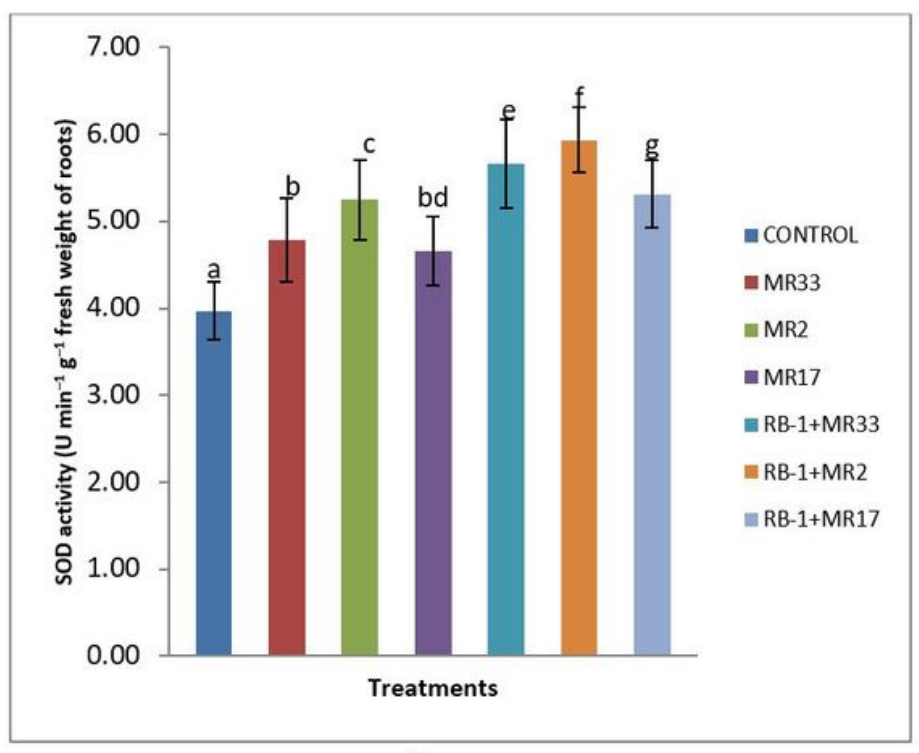

$4 a$

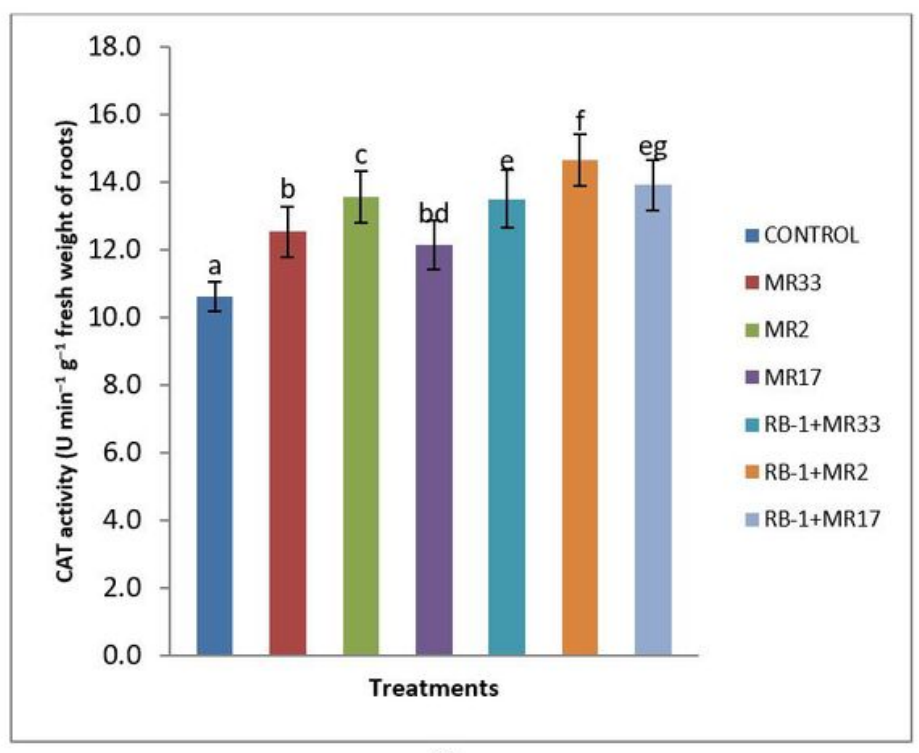

$4 b$

\section{Figure 4}

Effect of potential pesticide tolerant strains of Mesorhizobium sp. and rhizobacterium RB-1 on antioxidant enzymes activities in chickpea *Values presented are overall means of PBG and GPF2 chickpea varieties \pm SE (standard error). Means followed by dissimilar letter(s) on error bars are significantly different from each other at $P=\leq 0.05$ according to Tukey's least significance difference (LS 


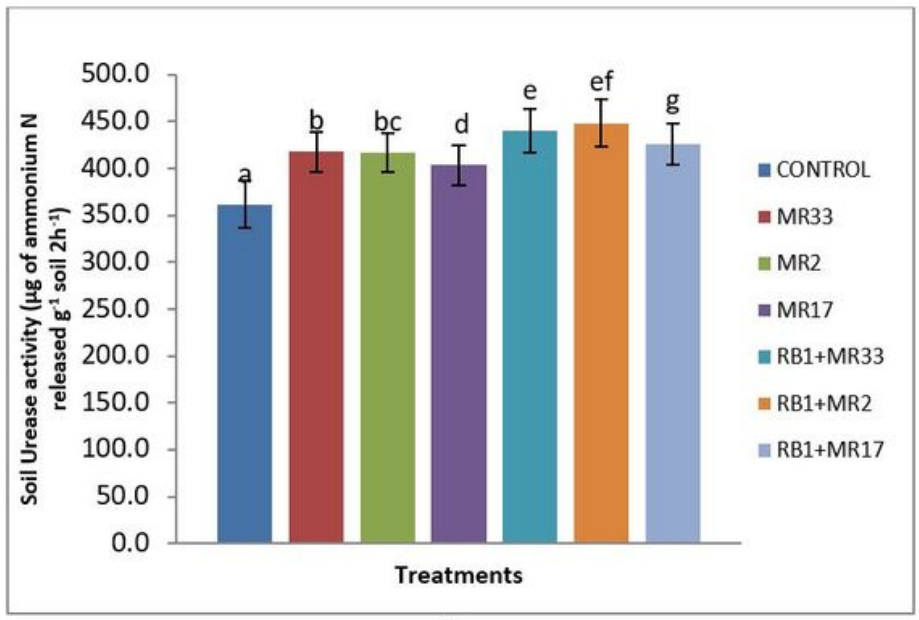

5 a

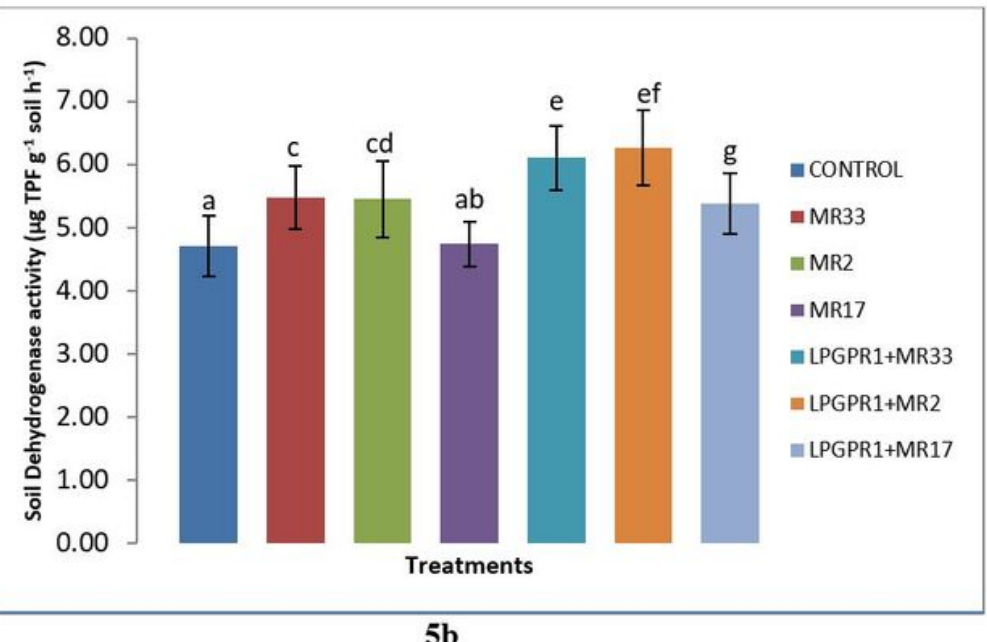

$5 b$

\section{Figure 5}

Effect of potential pesticide tolerant Mesorhizobium sp. strains and rhizobacterium strain RB-1 on soil enzyme activities in chickpea.

*Values presented are overall means of PBG and GPF2 chickpea varieties \pm SE (standard error). Means followed by dissimilar letter(s) on error bars are significantly different from each other at $P=\leq 0.05$ according to Tukey's least significance difference (LSD)

\section{Supplementary Files}

This is a list of supplementary files associated with this preprint. Click to download.

- PlatesPub1v2.doc 